\title{
Article \\ Diversity, Bioactivity Profiling and Untargeted Metabolomics of the Cultivable Gut Microbiota of Ciona intestinalis
}

\author{
Caroline Utermann ${ }^{1}\left(\mathbb{D}\right.$, Vivien A. Echelmeyer $^{1}$, Ernest Oppong-Danquah ${ }^{1}\left(\mathbb{D}\right.$, Martina Blümel ${ }^{1}(\mathbb{D}$ \\ and Deniz Tasdemir ${ }^{1,2}, *$ (D)
}

1 GEOMAR Centre for Marine Biotechnology (GEOMAR-Biotech), Research Unit Marine Natural Products Chemistry, GEOMAR Helmholtz Centre for Ocean Research Kiel, Am Kiel-Kanal 44, 24106 Kiel, Germany; cutermann@geomar.de (C.U.); vivienechelmeyer@web.de (V.A.E.); eoppong-danquah@geomar.de (E.O.-D.); mbluemel@geomar.de (M.B.)

2 Faculty of Mathematics and Natural Sciences, Kiel University, Christian-Albrechts-Platz 4, 24118 Kiel, Germany

* Correspondence: dtasdemir@geomar.de; Tel.: +49-431-600-4430

check for updates

Citation: Utermann, C.; Echelmeyer, V.A.; Oppong-Danquah, E.; Blümel, M.; Tasdemir, D. Diversity, Bioactivity Profiling and Untargeted Metabolomics of the Cultivable Gut Microbiota of Ciona intestinalis. Mar. Drugs 2021, 19, 6. https:/ /dx.doi.org/ 10.3390/md19010006

Received: 1 December 2020

Accepted: 22 December 2020

Published: 24 December 2020

Publisher's Note: MDPI stays neutral with regard to jurisdictional claims in published maps and institutional affiliations.

Copyright: () 2020 by the authors. Licensee MDPI, Basel, Switzerland. This article is an open access article distributed under the terms and conditions of the Creative Commons Attribution (CC BY) license (https: / / creativecommons.org/ licenses/by/4.0/).

\begin{abstract}
It is widely accepted that the commensal gut microbiota contributes to the health and wellbeing of its host. The solitary tunicate Ciona intestinalis emerges as a model organism for studying hostmicrobe interactions taking place in the gut, however, the potential of its gut-associated microbiota for marine biodiscovery remains unexploited. In this study, we set out to investigate the diversity, chemical space, and pharmacological potential of the gut-associated microbiota of $C$. intestinalis collected from the Baltic and North Seas. In a culture-based approach, we isolated 61 bacterial and 40 fungal strains affiliated to 33 different microbial genera, indicating a rich and diverse gut microbiota dominated by Gammaproteobacteria. In vitro screening of the crude microbial extracts indicated their antibacterial (64\% of extracts), anticancer (22\%), and/or antifungal (11\%) potential. Nine microbial crude extracts were prioritized for in-depth metabolome mining by a bioactivityand chemical diversity-based selection procedure. UPLC-MS/MS-based metabolomics combining automated (feature-based molecular networking and in silico dereplication) and manual approaches significantly improved the annotation rates. A high chemical diversity was detected where peptides and polyketides were the predominant classes. Many compounds remained unknown, including two putatively novel lipopeptides produced by a Trichoderma sp. strain. This is the first study assessing the chemical and pharmacological profile of the cultivable gut microbiota of $C$. intestinalis.
\end{abstract}

Keywords: tunicate; Ciona intestinalis; gut-associated microbiota; marine natural products; antimicrobial activity; anticancer activity; untargeted metabolomics; feature-based molecular networking; in silico MS/MS-based dereplication

\section{Introduction}

The animal gut is one of the most densely colonized microbial habitats representing a highly specialized internal ecosystem [1-3]. The commensal gut microbiota is known for contributing to the host's health and homeostasis by assisting, e.g., chemical defense, immunity, metabolic capacity, and digestion [1-3]. For instance, vertebrate-associated gut bacteria provide "colonization resistance" through, e.g., short-chain fatty acids to inhibit proliferation of pathogenic microorganisms such as Salmonella enterica [3,4]. Commensal gut bacteria also induce immune reactions by producing antimicrobial peptides or aid nutrient uptake by breaking down complex polysaccharides [3,4]. In the marine world, cultivable gut-associated bacteria of farmed fish, such as Vibrio sp., have been reported to inhibit common aquaculture pathogens, e.g., Vibrio anguillarum and Pasteurella piscicida [5,6]. The current evidence suggests that Vibrio spp. residing in the alimentary tract of the algafeeding sea urchin Strongylocentrotus spp. promote the animal's digestion by breaking down large algal polysaccharides such as alginates [7]. 
Marine microorganisms represent unparalleled resources for biodiscovery of compounds with great pharmaceutical application potential $[8,9]$. The majority of marine natural products (MNPs) discovered between 2014 and 2018 originate from bacteria or fungi [9]. Microorganisms associated with invertebrate hosts such as sponges and tunicates are promising resources for marine biodiscovery [8,10-12]. For example, ascidianassociated bacteria are prolific producers of antibiotics and anticancer drug leads $[11,13]$ including the anticancer drug Yondelis®produced by a gammaproteobacterial symbiont of the colonial sea squirt Ecteinascidia turbinata [14]. However, the gut microbiota of marine sessile animals has rarely been studied. The few available examples include the ascomycete fungi Aspergillus sp. and Letendraea sp., which were isolated from the gut of marine crustaceans [15-17]. They yielded novel cytotoxic aspochalazines [15,16] and the antiinflammatory polyketide phomopsiketone D [17], rendering the gut-associated microbiota of marine invertebrates as a valuable and underexploited source for MNP biodiscovery.

Host-associated microbial communities can be analyzed by culture-dependent and -independent methods. Culture-based studies capture only a small fraction of the actual microbiota $(\sim 0.001-1 \%)$, often select for easily culturable microorganisms, and, therefore, do not adequately reflect the microbial diversity $[18,19]$. In contrast, culture-independent approaches such as metagenomics and amplicon sequencing allow comprehensive description of the microbiota of interest, although the large majority of the detected microorganisms remains uncultivable [19]. Hence, comparison of the microbial diversity obtained by both methodologies often reveals a huge discrepancy [20], mainly due to the large fraction of uncultured microorganisms and the strong impact of the applied cultivation media on the culture-dependent microbial diversity $[18,19]$. Despite recent advances enabling, e.g., the access to compounds of yet uncultivable microorganisms via heterologous expression, marine biodiscovery studies still largely apply cultivation-dependent methods, mainly due to their high efficiency for bioactivity screening [21].

For decades, the sea vase Ciona spp. (chordate subphylum Tunicata) has served as a model organism for developmental biology, evolution, and chordate immunity [22,23]. Recently, C. intestinalis and C. robusta were introduced as model organisms for studying host-microbe interactions in animal gastrointestinal tracts, because they feature a compartmentalized gut resembling that of the vertebrates $[2,23,24]$. The gut of the filter feeder is in constant contact with millions of microbial cells posing a great challenge for the tunicate; on the one hand it must defend against pathogenic microorganisms, but at the same time allow colonization of commensals $[2,25]$. Initial studies have reported a few gut-associated Gammaproteobacteria (e.g., Shewanella sp. and Vibrio sp. $[23,26,27]$ ) and Ascomycota (e.g., Penicillium sp. and Trichoderma sp. [28]) from C. intestinalis and C. robusta. In line with this, amplicon sequencing of the bacterial community associated with $C$. intestinalis and C. robusta described a specific and diverse gut community dominated by Gammaproteobacteria [29], while culture-dependent and -independent studies on the tunic-associated microbiota of Ciona spp. revealed comparably high abundance of Alphaproteobacteria [30,31]. However, no information exists on chemical composition or biological activities of the gut-associated microbial community of $C$. intestinalis. This fact prompted us to isolate and study cultivable bacteria and fungi associated with the gut of C. intestinalis to explore their chemical machinery. Dissected guts of $C$. intestinalis sampled at two sites in Germany (Helgoland, North Sea and Kiel Fjord, Baltic Sea) yielded 61 bacterial and 40 fungal isolates. An initial bioactivity screening (antimicrobial and anticancer) and chemical profiling of the crude extracts of these microorganisms allowed selection of nine microbial extracts for LC-MS/MS-based untargeted metabolomics employing featurebased molecular networking (FBMN) [32], in silico [33] and manual dereplication tools. This study enabled us to prioritize two bacterial and three fungal strains for purification and characterization of their bioactive constituents in future. 


\section{Results}

\subsection{Cultivable Fraction of the Gut Microbiota of C. intestinalis}

Application of six different cultivation media led to 61 bacterial and 40 fungal isolates from the gut of $C$. intestinalis sampled in Helgoland $(\mathrm{H})$ and Kiel Fjord (K; Figure 1a, Table S1). The number of bacterial isolates was much higher in the Baltic Sea samples than in the North Sea samples (H: 24, K: 37). However, samples from Kiel Fjord (Baltic Sea) and the North Sea island Helgoland yielded similar numbers of fungal isolates $(\mathrm{H}$ : $18, \mathrm{~K}: 22)$. As expected, the applied isolation media had a remarkable influence on the number of isolated microorganisms (Figure 1b). Isolation on modified Wickerham medium (WSP) yielded the highest number of isolates (32, compared to other media: 7-24 isolates). Bacterial isolates derived mainly from Marine Broth (MB; $26 \%$ ) and tryptic soy broth (TSB; $31 \%$ ) media, while gut-associated fungal strains were mainly obtained from WSP medium (20 isolates; other media: 1-7 fungal isolates). Similar to our previous study that reported the tunic-associated microbiota of $C$. intestinalis [31], we used media resembling the natural habitat of the isolates, i.e., media adjusted to Baltic (CB) or North Sea (CN) salinity containing $C$. intestinalis powder. They yielded many microbial strains $(n=20)$, and the microbial genera Acrostalagmus, Arthopyrenia, Cordyceps, and Sporosarcina were exclusively isolated from these media. Accordingly, the $C$. intestinalis media CB and CN proved valuable for isolating a diverse tunicate-associated microbiota.

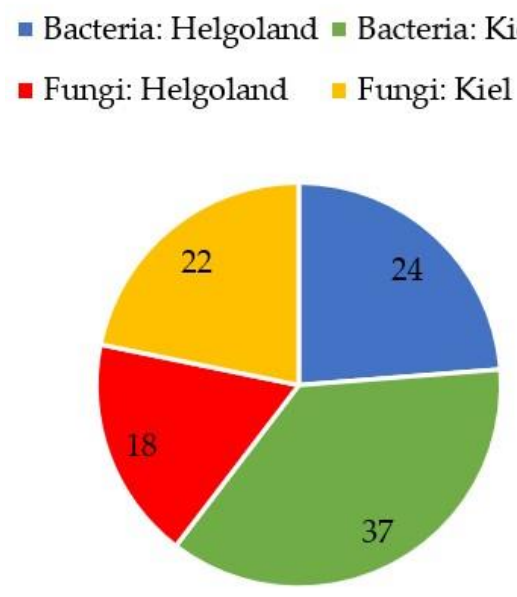

(a)

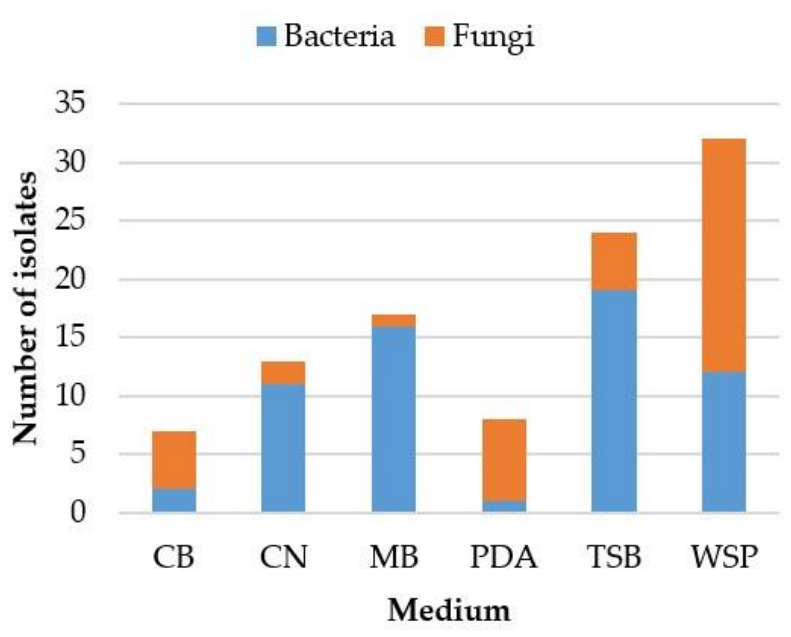

(b)

Figure 1. Distribution of bacterial $(n=61)$ and fungal $(n=40)$ isolates deriving from the gut of Ciona intestinalis sampled in the North (Helgoland) and Baltic Seas (Kiel Fjord). Numbers of bacterial and fungal isolates are displayed for (a) the two different sampling sites and (b) with respect to the used isolation media. CB/CN: C. intestinalis media adjusted to Baltic (CB) or North Sea (CN) salinity; MB: Marine Broth; PDA: potato dextrose agar; TSB: trypticase soy broth; WSP: modified Wickerham medium.

Compositionally, the cultivable microbiota was affiliated to three bacterial (Actinobacteria, Firmicutes, Proteobacteria) and two fungal phyla (Ascomycota, Mucoromycota; Table S1). Sanger sequencing allowed identification of all but one isolate to species (31 isolates) or genus (69 isolates) level. The gut-associated bacterial community was dominated by Shewanella sp. (H: 7 isolates, K: 6 isolates) and Vibrio sp. (H: 6 isolates, K: 8 isolates; Figure 2a). Out of 13 bacterial genera, six were exclusively found in Baltic C. intestinalis gut samples (Klebsiella, Micromonospora, Nocardiopsis, Pseudomonas, Rhodococcus, and Sporosarcina), while only two were exclusive to Helgoland (Escherichia and Ruegeria). Moreover, Bacillus sp. showed higher abundance in Kiel (9 isolates) compared to samples from Helgoland ( 2 isolates). Penicillium was the predominant fungal genus with four (from $\mathrm{H}$ ) or five isolates (from $\mathrm{K}$ ), respectively (Figure $2 \mathrm{~b}$ ). Out of 20 detected fungal genera, only 
Fusarium, Galactomyces, Penicillium, and Trichoderma were common to both locations indicating a differential fungal diversity of the gut of $C$. intestinalis collected from Helgoland and Kiel Fjord. Helgoland-exclusive fungal genera included, e.g., Arthrinium sp. (2 isolates) and Aspergillus sp. (3 isolates). The gut of Baltic C. intestinalis delivered 10 exclusive fungal genera such as Mucor sp. (2 isolates), Purpureocillium sp. (3 isolates), and Sarocladium sp. ( 2 isolates).

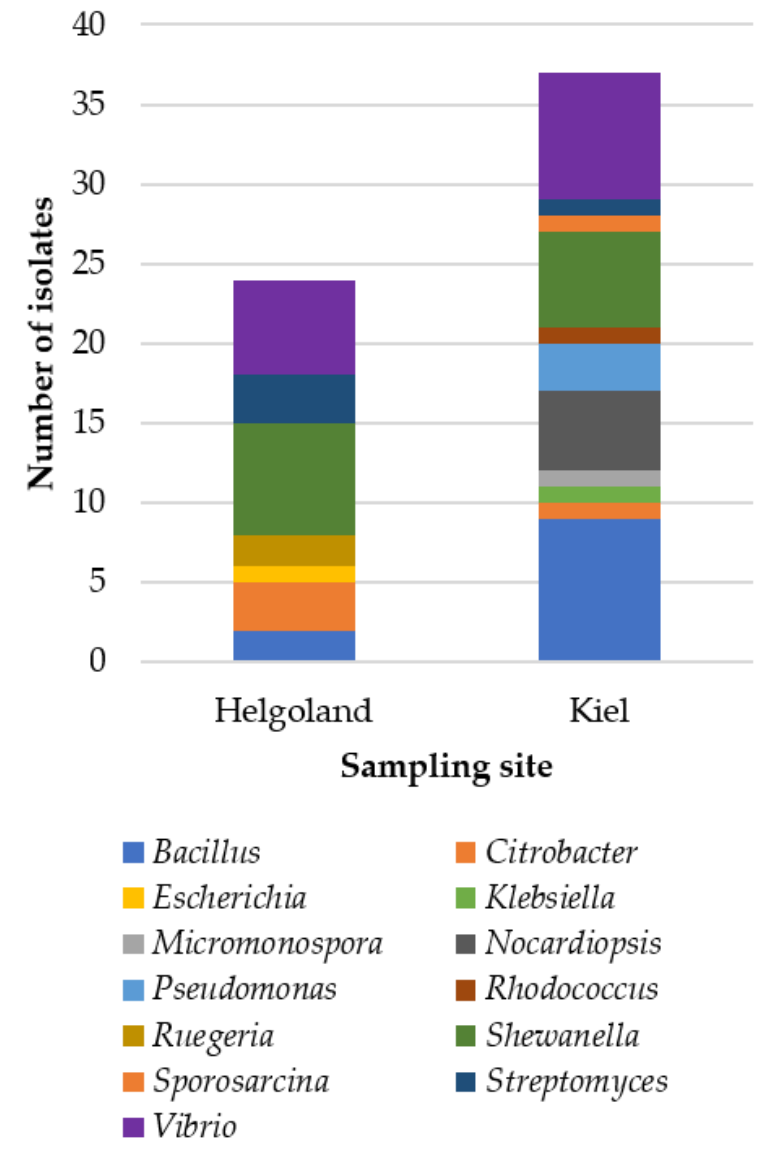

(a)

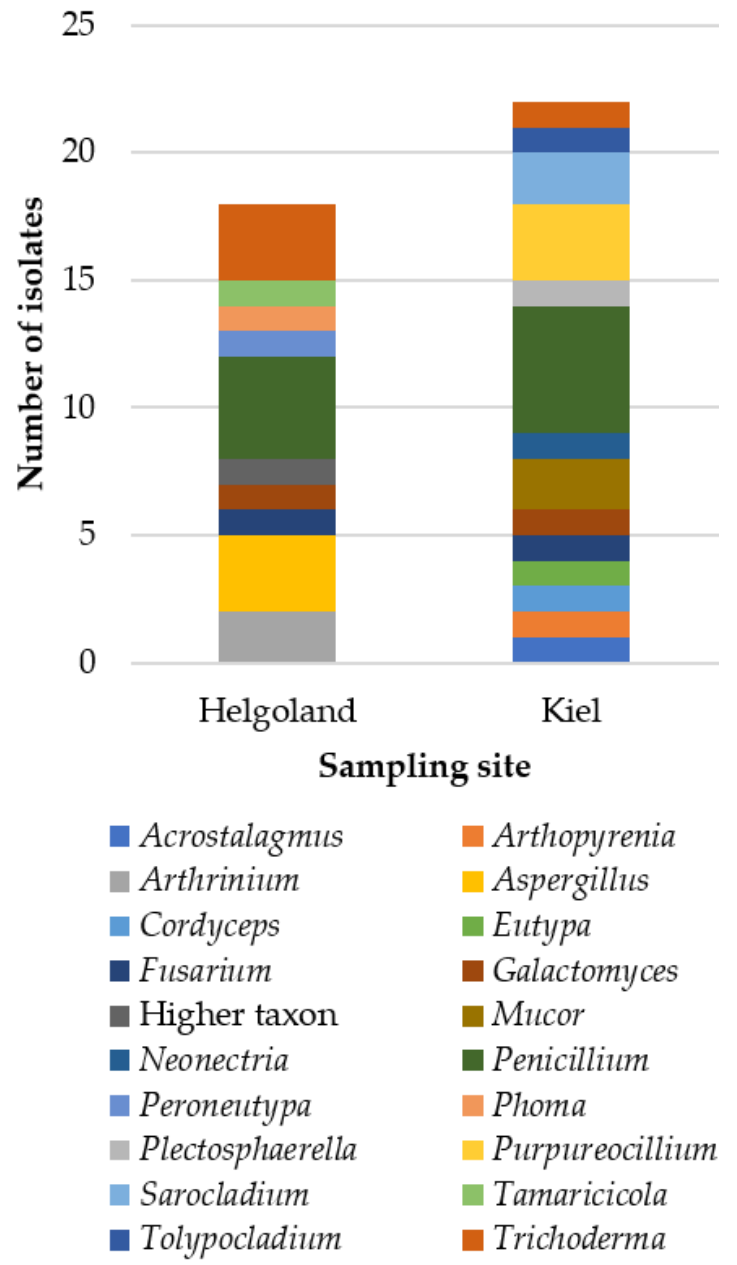

(b)

Figure 2. Diversity of (a) bacterial and (b) fungal isolates associated with the gut of $C$. intestinalis at genus level. The designation "Higher taxon" refers to isolate CHG49 (only identified to family level, Pleosporaceae).

\subsection{Biological Activities of Gut-Derived Microbial Extracts}

To assess the biotechnological potential of the gut-associated microbiota, bacterial isolates were cultured on the agar media glucose-yeast-malt (GYM) and Marine Broth (MB), while fungi were grown on solid casamino acids-glucose (CAG) and potato dextrose agar (PDA) media. Extracts received identification codes referring to the host organism C. intestinalis $(\mathrm{C})$, the sampling site $(\mathrm{H}$ or $\mathrm{K})$, the origin of the microbial isolates (gut, $\mathrm{G})$, the respective strain number, and cultivation medium (CAG, GYM, MB or PDA). For example, CHG2-MB is the Marine Broth extract of strain 2 that was isolated from the gut of $C$. intestinalis sampled in Helgoland.

In vitro bioactivities were determined for 103 microbial crude extracts against eight human microbial pathogens including the ESKAPE panel (see Section 4.4.), Candida albicans, and Cryptococcus neoformans, and against four cancer cell lines. A total of 68 extracts 
(reflecting $66 \%$ ) were active at a bioactivity threshold of $\geq 80 \%$ inhibition (= highly active, test concentration of $100 \mu \mathrm{g} / \mathrm{mL}$ ) in at least one assay (Table S2). Most frequently, activity was observed against the Gram-positive bacterial pathogens methicillin-resistant Staphylococcus aureus (MRSA; 62\%) and Enterococcus faecium (50\%; Figure 3, Table S2). Notably, twelve crude extracts derived from the fungi Acrostalagmus luteoalbus (CKG66-CAG), Galactomyces candidum (CKG25-CAG, -PDA), Penicillium sp. (CHG25-CAG, -PDA, CHG35CAG, -PDA, CKG23-CAG, -PDA, CKG63-PDA), and Pleosporaceae sp. (CHG49-CAG, -PDA) inhibited the growth of at least one Gram-negative test strain. The Penicillium sp. extract CHG 25 -CAG showed $98 \%$ to $100 \%$ growth inhibitory activity against all four Gram-negative bacterial pathogens (Acinetobacter baumannii, Escherichia coli, Klebsiella pneumoniae, and Pseudomonas aeruginosa). Eleven extracts exhibited antifungal activity against Candida albicans and/or Cryptococcus neoformans. Among them, three extracts, namely, extracts produced by Streptomyces sp. (CHG48-GYM) and Trichoderma sp. (CHG34-PDA and CKG62-PDA), were active against both fungal pathogens. Anticancer activity was detected in 23 crude extracts. Proliferation of all four cancer cell lines was inhibited by five bacterial (Micromonospora sp. CKG20-GYM; Nocardiopsis prasina CKG58-GYM; Streptomyces sp. CHG40-GYM, CHG48-GYM, CHG64-GYM) as well as ten fungal extracts (A. luteoalbus CKG66-CAG; G. candidum CKG25-CAG, -PDA; Penicillium sp. CHG25-CAG, -PDA, CKG23CAG; Pleosporaceae CHG49-CAG; and Trichoderma sp. CHG34-CAG, -PDA, CKG62-PDA). Five extracts showed somewhat narrow spectrum anticancer activity, for example, Fusarium sp. extract CHG38-CAG strongly inhibited the growth of the human melanoma cells (A375, $98 \%$ ) and colon cancer cells (HCT116, 93\%), but was only moderately or poorly active against the lung cancer (A549, 65\%) and breast cancer cells (MB231, 40\%).

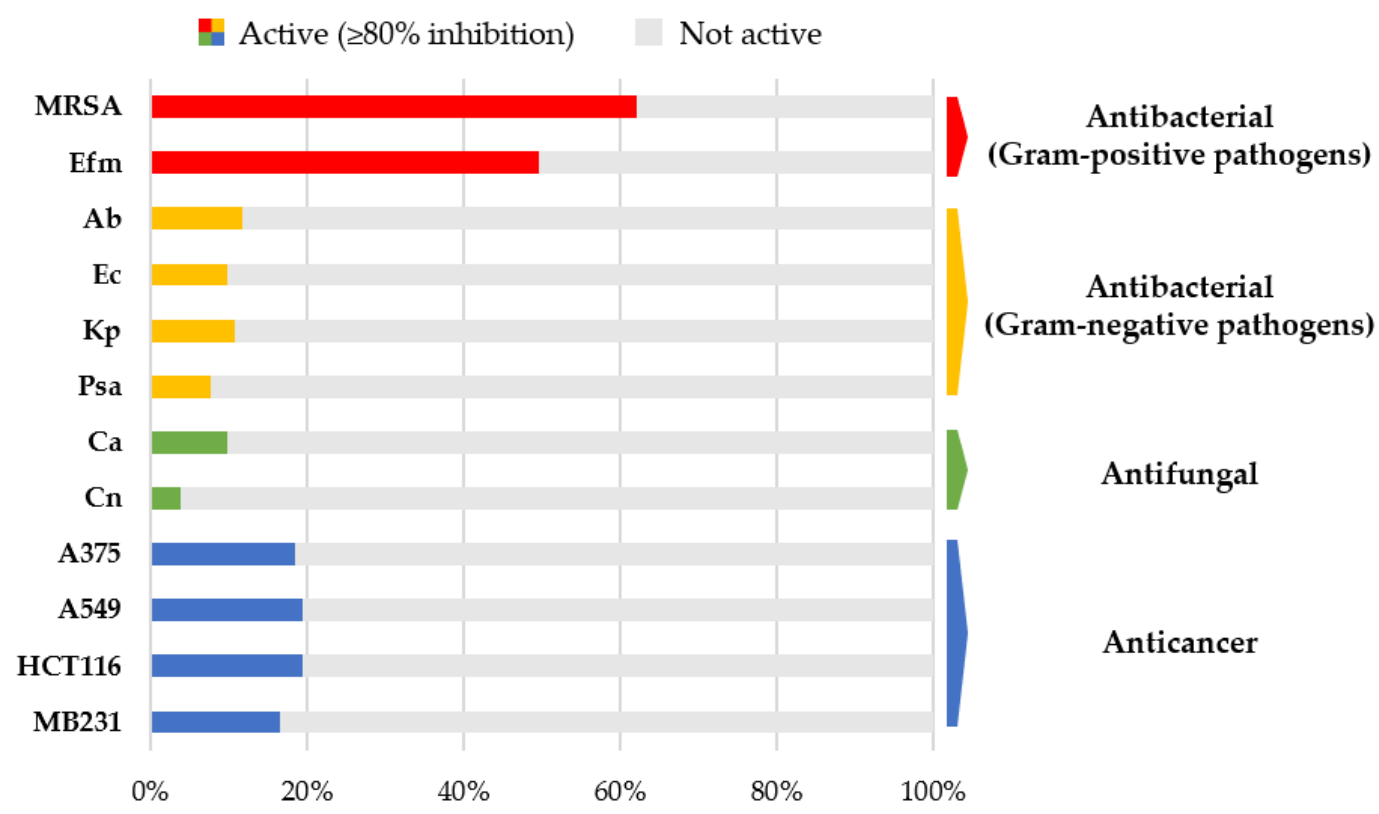

Figure 3. Antimicrobial and anticancer activities of 103 microbial crude extracts. Extracts were classified as active when showing inhibitory activity $\geq 80 \%$ at a test concentration of $100 \mu \mathrm{g} / \mathrm{mL}$. Activities were determined against Gram-positive bacterial pathogens (red; MRSA: methicillin-resistant Staphylococcus aureus, Efm: Enterococcus faecium), Gram-negative bacterial pathogens (yellow; Ab: Acinetobacter baumannii, Ec: Escherichia coli, Kp: Klebsiella pneumoniae, Psa: Pseudomonas aeruginosa), fungal pathogens (green; Ca: Candida albicans, Cn: Cryptococcus neoformans) and cancer cell lines (blue; A375: malignant melanoma, A549: lung carcinoma, HCT116: colon cancer, MB231: breast cancer).

\subsection{Bioactivity- and Metabolome-Based Selection of Microbial Extracts}

In order to prioritize the most promising candidates out of 68 active crude extracts, we applied a two-step selection approach. In the first step, all extracts with (i) high antimi- 
crobial activity ( $\geq 80 \%$ inhibition) against at least one bacterial and one fungal pathogen, or (ii) high anticancer activity ( $\geq 80 \%$ inhibition) against at least one cancer cell line, or (iii) both, high antimicrobial and anticancer activity ( $\geq 80 \%$ inhibition), were selected. This approach allowed us to prioritize 26 extracts, including eight bacterial extracts obtained from Bacillus sp. (CKG24-GYM), Micromonospora sp. (CKG20-GYM), N. prasina (CKG58GYM), Pseudomonas anguilliseptica (CKG38-GYM, -MB), and Streptomyces sp. (CHG40-GYM, CHG48-GYM, CHG64-GYM), as well as 18 fungal extracts originating from A. luteoalbus (CKG66-CAG), Fusarium sp. (CHG38-CAG, -PDA, CKG32-CAG), G. candidum (CKG25CAG,-PDA), Penicillium sp. (CHG25-CAG,-PDA, CHG35-CAG, -PDA, CKG23-CAG, -PDA, CKG63-PDA), Pleosporaceae (CHG49-CAG, -PDA), and Trichoderma sp. (CHG34-CAG, -PDA, CKG62-PDA).

In the second step, metabolite profiling by an untargeted UPLC-MS/MS-based approach was applied to the 26 pre-selected extracts in order to detect those with the richest and most diverse chemistry. Pre-processed MS/MS data were converted into peak lists ( $m / z$ value, retention time, intensity) to generate PCoA (Principal Coordinates Analysis) plots reflecting the chemical distinctiveness of the bacterial (Figure 4) and fungal extracts (Figure 5).

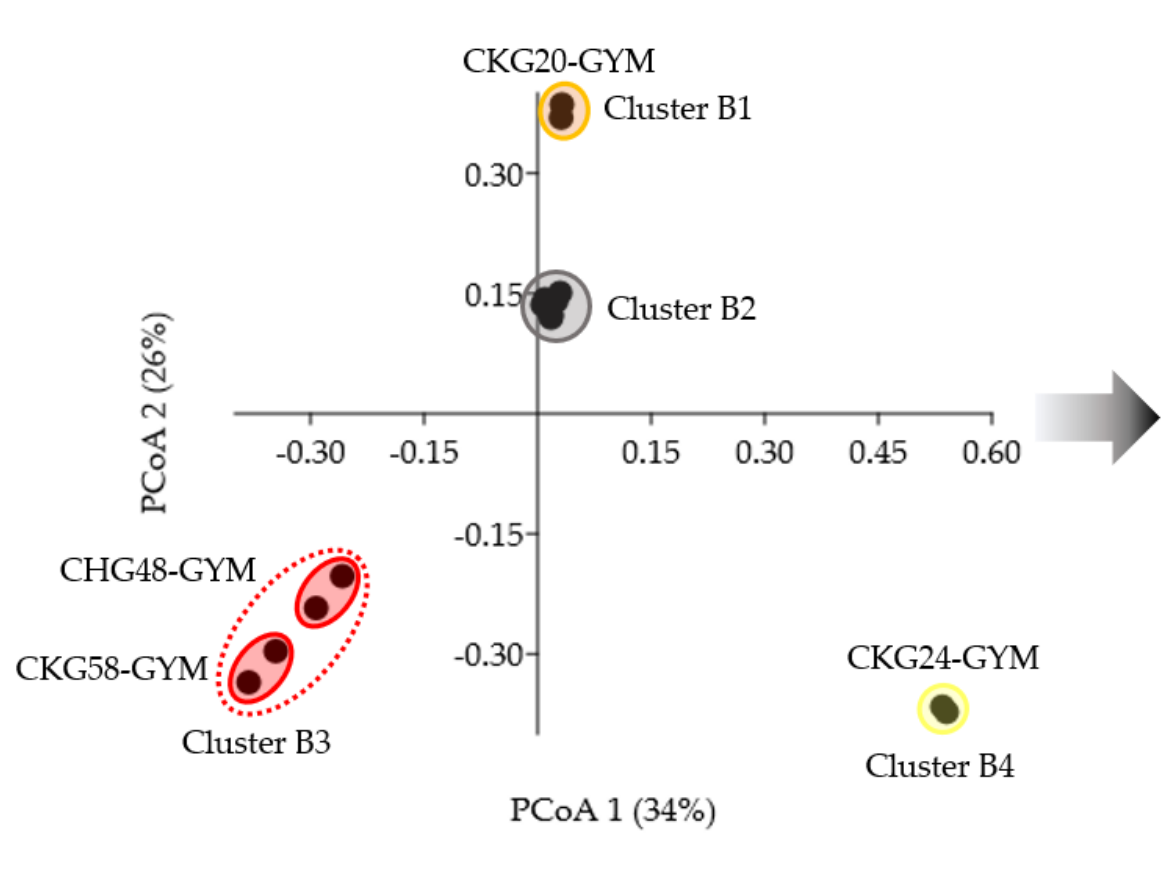

(a)

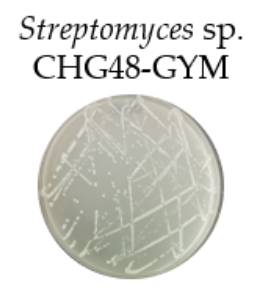

Micromonospora sp. CKG20-GYM

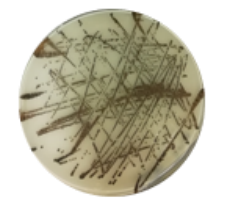

Bacillus sp. CKG24-GYM

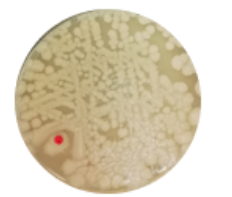

(b)

Figure 4. UPLC-MS/MS-based selection of bacterial extracts for in-depth metabolomics. (a) PCoA (Principal Coordinates Analysis) plot of eight pre-selected bioactive extracts. Cluster B2 includes extracts produced by Pseudomonas anguilliseptica (CKG38-glucose-yeast-malt (GYM) and-MB) and Streptomyces sp. (CHG40-GYM and CHG64-GYM). (b) Solid cultures of three bacterial extracts prioritized for further chemical investigations. 


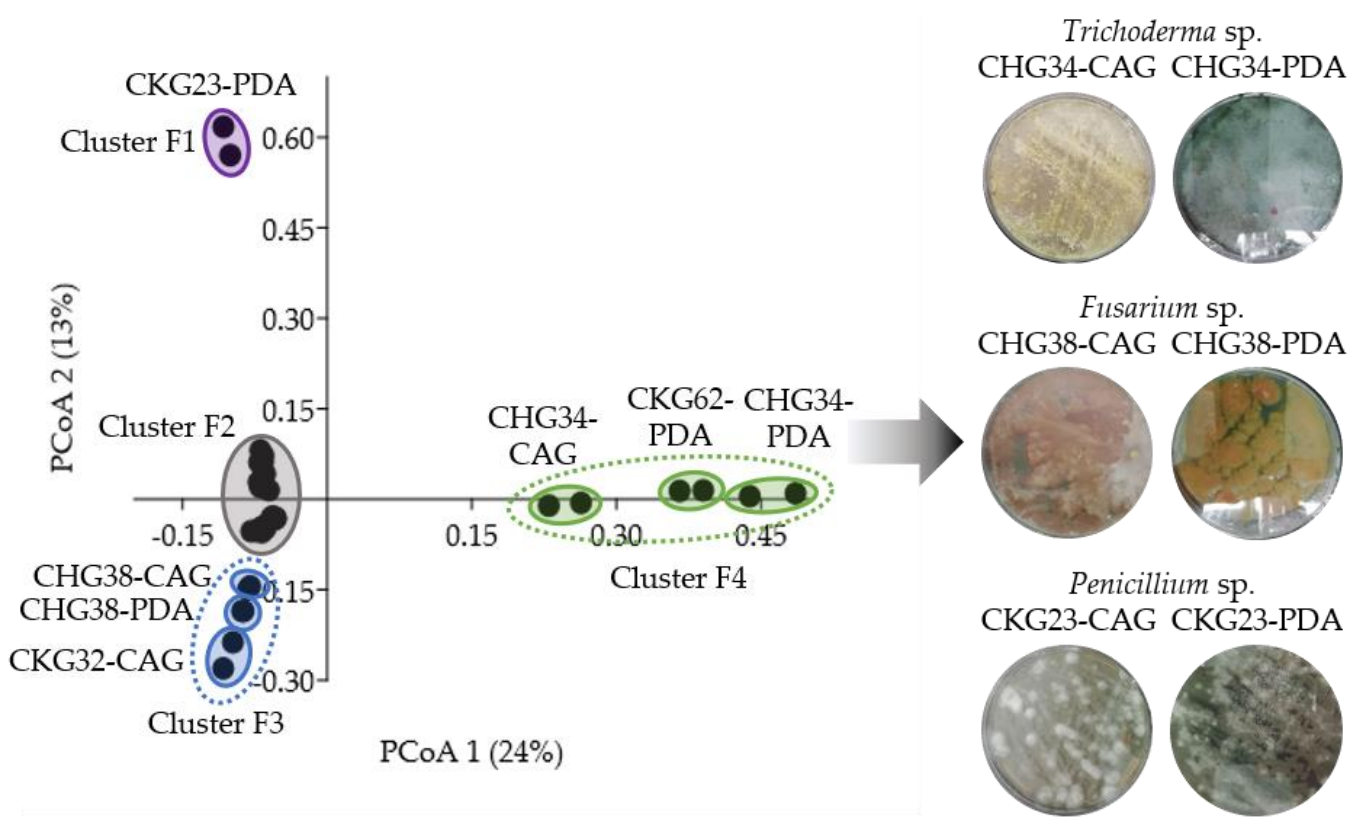

(a)

(b)

Figure 5. UPLC-MS/MS-based selection of fungal extracts for in-depth metabolomics. (a) PCoA plot of 18 pre-selected bioactive extracts. Cluster F2 includes extracts produced by Acrostalagmus luteoalbus (CKG66-casamino acids-glucose (CAG)), Galactomyces candidum (CKG25-CAG and -PDA), Penicillium sp. (CHG25-CAG and -PDA, CHG35-CAG and -PDA, CKG23-CAG, and CKG63-PDA), and Pleosporaceae sp. (CHG49-CAG and -PDA). (b) Solid cultures of six fungal extracts prioritized for further chemical investigations.

The PCoA plot of the eight pre-selected bacterial extracts clustered into four groups (B1-B4; Figure 4a). Three clusters, i.e., B1, B3, and B4, showed significant differences in their chemical profiles compared to cluster $B 2(R=1, p<0.05$; Table S3). The chemically distinct extracts in clusters B1, B3, and B4 had a higher number of detected peaks (80-110) compared to those clustering as B2 (9-66 peaks). Micromonospora sp. extract CKG20GYM and Bacillus sp. extract CKG24-GYM clustered separately as B1 and B4, respectively. Accordingly, both were selected for further analysis. Cluster B3 was formed by two actinobacterial extracts with similar chemistry, namely, N. prasina extract CKG58-GYM and Streptomyces sp. extract CHG48-GYM. The latter extract was selected from cluster B3, since it showed higher bioactivity against C. neoformans ( $100 \%$ inhibition at $100 \mu \mathrm{g} / \mathrm{mL}$; Table S2), and displayed, with 103 peaks, a more distinct metabolome than the other Streptomyces extracts CHG40-GYM (61 peaks) and CHG64-GYM (66 peaks) in cluster B2 (Figure S1). Hence, metabolite profiling aided the prioritization of the three bacterial extracts CHG48GYM (Streptomyces sp.), CKG20-GYM (Micromonospora sp.), and CKG24-GYM (Bacillus sp.) for subsequent metabolomics studies (Figure $4 b$ ).

The same process was applied to 18 bioactive fungal extracts resulting in four different clusters in the PCoA plot (clusters F1-F4; Figure 5a). Extracts of three clusters (F1, F3, and F4) had significantly different metabolite profiles compared to extracts from cluster F2 $(R=0.68-1, p<0.01$; Table S4). Cluster F1 contained only one extract (Penicillium sp. CKG23-PDA), which was selected due to its strikingly different chemical profile. Cluster F3 consisted of three extracts obtained from two Fusarium sp. strains CHG38 (CAG and PDA) and CKG32 (PDA). From those, we selected strain CHG38 (CAG and PDA) because of the additional antifungal and anticancer bioactivities observed for the extract CHG38-CAG (96\% inhibition against C. neoformans, 98\% and 93\% inhibition against cancer cell lines A375 and HCT116, respectively; Table S2). Similarly, cluster F4 contained in total three extracts from two Trichoderma sp. isolates CHG34 (CAG and PDA) and CKG62 (PDA). Revisiting these extracts' bioactivities led to the selection of Trichoderma sp. strain CHG34 (CAG and PDA), as its PDA extract showed in addition strong antibacterial (MRSA, 94\%) and 
antifungal (C. neoformans, 92\%) activities (Table S2). In addition, we added Penicillium sp. CKG23-CAG (cluster F2) to the analysis pipeline, although it did not fulfill the chemical distinctiveness criterion. It shared only few metabolites with the PDA extract from the same Penicillium sp. strain (CKG23-PDA), which clustered in F1. Therefore, we aimed to analyze, comparatively, these two chemically different extracts (CKG23-CAG and CKG23-PDA) produced by the same Penicillium sp. strain. In total, six fungal extracts were prioritized for further chemical analyses, namely CHG34-CAG and -PDA (Trichoderma sp.), CHG38-CAG and -PDA (Fusarium sp.), and CKG23-CAG and -PDA (Penicillium sp.; Figure 5b). This sums up to a total of nine microbial crude extracts for subsequent in-depth metabolomic analyses.

\section{4. $I C_{50}$ Determinations of Prioritized Microbial Extracts}

All nine prioritized extracts were subjected to $\mathrm{IC}_{50}$ determinations (half maximal inhibitory concentration) against bacterial and fungal human pathogens (Table 1) as well as cancer cell lines (Table 2). The lowest $\mathrm{IC}_{50}$ values against the Gram-positive test strains MRSA and E. faecium were obtained for Streptomyces sp. CHG48-GYM, Micromonospora sp. CKG20-GYM, Bacillus sp. CKG24-GYM, and Fusarium sp. CHG38-CAG (Table 1). Notably, the anti-MRSA potency of Bacillus sp. extract CKG24-GYM (IC 50 value $0.4 \mu \mathrm{g} / \mathrm{mL}$ ) was about eight times higher than the positive control chloramphenicol $\left(\mathrm{IC}_{50} 3.1 \mu \mathrm{g} / \mathrm{mL}\right)$. Another very potent bacterium was Micromonospora sp. grown in GYM medium (CKG20GYM), which showed superior activity $\left(\mathrm{IC}_{50} 0.1 \mu \mathrm{g} / \mathrm{mL}\right.$ ) to the reference antibiotic ampicillin $\left(\mathrm{IC}_{50} 0.4 \mu \mathrm{g} / \mathrm{mL}\right.$ ) against E. faecium. Only Penicillium sp. extracts CKG23-CAG and -PDA showed inhibitory activity against the four Gram-negative bacterial test strains A. baumannii, E. coli, K. pneumoniae, and P. aeruginosa. Notably, $\mathrm{IC}_{50}$ values of the CAG extract were more potent $\left(\mathrm{IC}_{50}\right.$ values between 4.9 and $15.8 \mu \mathrm{g} / \mathrm{mL}$ ) than those of the PDA extract ( $\mathrm{IC}_{50}$ values between 31.4 and $42.6 \mu \mathrm{g} / \mathrm{mL}$ ). Concerning antifungal activity, the lowest $\mathrm{IC}_{50}$ value against $C$. albicans was exerted by the PDA extract of Trichoderma sp. isolate CHG34 ( $\mathrm{IC}_{50}$ value $3.7 \mu \mathrm{g} / \mathrm{mL}$ ). With an $\mathrm{IC}_{50}$ value of $13.1 \mu \mathrm{g} / \mathrm{mL}$, Streptomyces sp. CHG48-GYM emerged as the most potent extract towards the yeast-like pathogen C. neoformans.

When tested against cancer cell lines, all extracts showed inhibitory activity against at least one cancer cell line ( $\mathrm{IC}_{50}$ values between 0.02 and $92.3 \mu \mathrm{g} / \mathrm{mL}$; Table 2). Streptomyces sp. extract CHG48-GYM showed the strongest anticancer activity with an $\mathrm{IC}_{50}$ value $0.02 \mu \mathrm{g} / \mathrm{mL}$ against the lung carcinoma cell line A549, which was much lower compared to the positive control ( $\mathrm{IC}_{50} 1.3 \mu \mathrm{g} / \mathrm{mL}$ ). Micromonospora sp. extract CKG20-GYM showed potent in vitro cytotoxicity against all four cancer cell lines $\left(\mathrm{IC}_{50}\right.$ values between 0.8 and $1.6 \mu \mathrm{g} / \mathrm{mL}$ ). Among the fungal extracts, Penicillium sp. extract CKG23-CAG exerted the strongest antiproliferative effects $\left(\mathrm{IC}_{50}\right.$ values between 2.0 and $\left.8.5 \mu \mathrm{g} / \mathrm{mL}\right)$.

Table 1. $\mathrm{IC}_{50}$ values (in $\mu \mathrm{g} / \mathrm{mL}$ ) of selected extracts against eight microbial human pathogens. MRSA: methicillin-resistant S. aureus; Efm: E. faecium; Ab: A. baumannii; Ec: E. coli; Kp: K. pneumoniae; Psa: P. aeruginosa; Ca: C. albicans; Cn: C. neoformans. Positive controls: chloramphenicol (MRSA, Ec, Kp), ampicillin (Efm), doxycycline (Ab), polymyxin B (Psa), nystatin (Ca), and amphotericin (Cn).

\begin{tabular}{cccccccccc}
\hline Extract & Identification & MRSA & Efm & Ab & Ec & Kp & Psa & Ca & Cn \\
\hline CHG48-GYM & Streptomyces sp. & 5.0 & 4.5 & $>100$ & $>100$ & $>100$ & $>100$ & 12.9 & 13.1 \\
CKG20-GYM & Micromonospora sp. & 10.3 & 0.1 & $>100$ & $>100$ & $>100$ & $>100$ & $>100$ & $>100$ \\
CKG24-GYM & Bacillus sp. & 0.4 & 2.0 & $>100$ & $>100$ & $>100$ & $>100$ & $>100$ & 17.1 \\
CHG34-CAG & Trichoderma sp. & $>100$ & $>100$ & $>100$ & $>100$ & $>100$ & $>100$ & $>100$ & $>100$ \\
CHG34-PDA & Trichoderma sp. & 36.8 & 32.0 & $>100$ & $>100$ & $>100$ & $>100$ & 3.7 & 58.8 \\
CHG38-CAG & Fusarium sp. & 4.0 & 3.0 & $>100$ & $>100$ & $>100$ & $>100$ & 9.9 & 20.9 \\
CHG38-PDA & Fusarium sp. & 10.8 & 7.6 & $>100$ & $>100$ & $>100$ & $>100$ & 11.4 & $>100$ \\
CKG23-CAG & Penicillium sp. & 19.8 & 29.8 & 4.9 & 15.6 & 8.9 & 15.8 & $>100$ & $>100$ \\
CKG23-PDA & Penicillium sp. & 8.3 & 18.1 & 42.5 & 41.0 & 31.4 & 42.6 & $>100$ & $>100$ \\
Positive control & & 3.1 & 0.4 & 0.02 & 1.4 & 0.4 & 0.4 & 1.3 & 0.1 \\
\hline
\end{tabular}


Table 2. $\mathrm{IC}_{50}$ values $(\mu \mathrm{g} / \mathrm{mL})$ of selected extracts against four cancer cell lines. A375: malignant melanoma cell line; A549: lung carcinoma cell line; HCT116: colon cancer cell line; MB231: breast cancer cell line. Positive control: doxorubicin.

\begin{tabular}{cccccc}
\hline Extract & Identification & A375 & A549 & HCT116 & MB231 \\
\hline CHG48-GYM & Streptomyces sp. & 5.8 & 0.02 & 21.4 & 22.1 \\
CKG20-GYM & Micromonospora sp. & 0.8 & 1.6 & 1.3 & 1.4 \\
CKG24-GYM & Bacillus sp. & 70.7 & 67.9 & 86.6 & $>100$ \\
CHG34-CAG & Trichoderma sp. & 67.9 & 70.1 & 71.9 & 69.7 \\
CHG34-PDA & Trichoderma sp. & 19.3 & 31.1 & 24.1 & 32.8 \\
CHG38-CAG & Fusarium sp. & 35.9 & 70.1 & 62.1 & $>100$ \\
CHG38-PDA & Fusarium sp. & 92.3 & $>100$ & $>100$ & $>100$ \\
CKG23-CAG & Penicillium sp. & 2.0 & 4.9 & 8.5 & 2.5 \\
CKG23-PDA & Penicillium sp. & 5.2 & 17.5 & 27.6 & 9.0 \\
Positive control & & 0.8 & 1.3 & 13.3 & 2.3 \\
\hline
\end{tabular}

\subsection{Feature-Based Molecular Networking and Dereplication of Nine Prioritized Microbial Extracts}

We comparatively analyzed the metabolome of the nine prioritized extracts by an integrated dereplication strategy combining FBMN, in silico dereplication tools, and manual approaches. Chemical structures of the putatively annotated metabolites, dereplication tables, and generated FBMNs are shown in the Supplementary Figures S2-S8 and Tables S5-S10.

Global metabolome analyses of three selected bacterial extracts, Streptomyces sp. CHG48-GYM, Micromonospora sp. CKG20-GYM, and Bacillus sp. CKG24-GYM, revealed a high metabolite diversity with a total of 220 nodes (ions) organized into 35 molecular clusters (Figure 6). Out of the 35 molecular clusters, 21 were putatively annotated as acetamide derivatives, cyclic peptides (including lipo- and depsipeptides), diterpenoid glycosides, glycerophospholipids, isocoumarin derivatives, nonactic acid polyketides, oxazolidone alkaloids, phenazine alkaloids, and polyketide glycosides. The global FBMN was dominated by several types of cyclic peptides, produced by Micromonospora sp. (CKG20-GYM) and Bacillus sp. (CKG24G-GYM). The Bacillus sp. extract CKG24-GYM exhibited the richest metabolite diversity (89 nodes), followed by Streptomyces sp. CHG48-GYM (73 nodes), and Micromonospora sp. CKG20-GYM (61 nodes). The chemical diversity of Bacillus sp. extract CKG24-GYM was also reflected in the number of exclusive clusters (15 exclusive molecular clusters) compared to CHG48-GYM (11 exclusive molecular clusters) and CKG20GYM (6 exclusive molecular clusters). Most molecular clusters (91\%) in the composite FBMN were exclusive to one bacterial extract. Shared metabolites, only detected in two molecular clusters, remained unannotated.

In-depth chemical investigations of Streptomyces sp. extract CHG48-GYM led to the putative annotation of the alkaloids streptazolin (2) and streptenol E (3), the diterpenoid glycoside platensimycin B4 (6), the linear polyketide alpiniamide A (7), and four nonactic acid polyketides $(\mathbf{1 2}, \mathbf{1 4}, \mathbf{1 8}, \mathbf{3 3}$; Figure 6, Figure S3 and Table S5). Nonactic-acid-type polyketides formed the two largest clusters in the molecular network of Streptomyces sp., representing protonated $\left([\mathrm{M}+\mathrm{H}]^{+}\right)$and sodiated adducts $\left([\mathrm{M}+\mathrm{Na}]^{+}\right)$detected from this chemical family (Figure S3). FBMN-based dereplication led to the putative annotation of, e.g., nonactic acid polyketides. MS/MS library spectra of bonactin (14) and homononactyl homononactate (18) deposited at the Global Natural Products Social Molecular Networking platform (GNPS, [34]) revealed a precise match with the MS/MS spectra detected for $m / z 401.2540[\mathrm{M}+\mathrm{H}]^{+}(\mathbf{1 4})$ and $m / z 415.2702[\mathrm{M}+\mathrm{H}]^{+}(\mathbf{1 8}$; Figures S9 and S10). This aided manual identification of nonactyl nonactoate (12) and nonactin (33). Nevertheless, most compounds and clusters could not be linked to any known chemical entity, and, therefore, many compounds (76\%) in Streptomyces sp. extract CHG48-GYM remained unknown. 


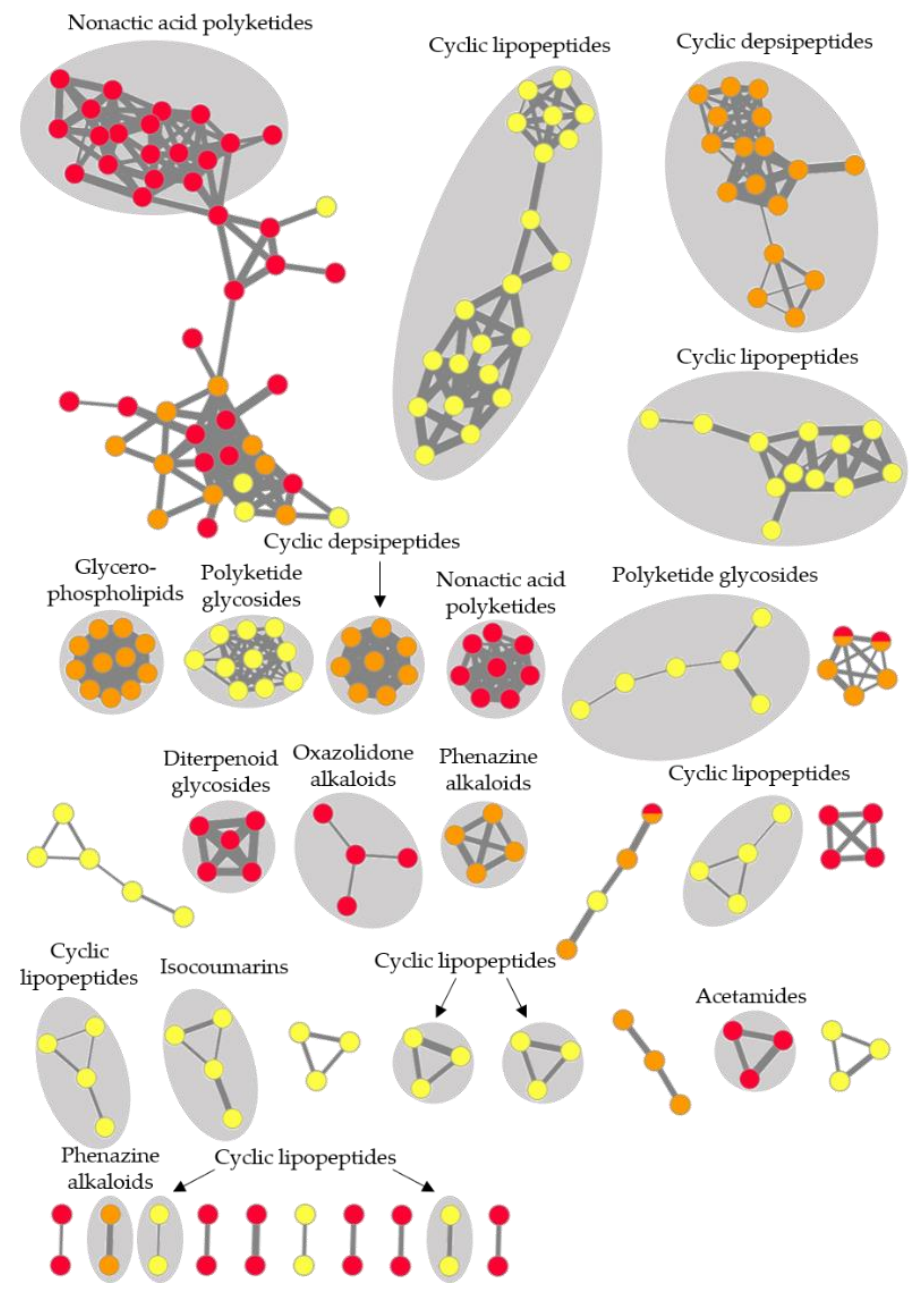

Figure 6. UPLC-MS/MS-based metabolome of three bacterial extracts. The feature-based molecular network (FBMN) displays only clusters containing $\geq 2$ nodes. The width of edges represents the cosine similarity between 2 nodes. Nodes are color-coded by the respective extract: red: Streptomyces sp. extract CHG48-GYM; orange: Micromonospora sp. extract CKG20-GYM; yellow: Bacillus sp. extract CKG24-GYM.

The Micromonospora sp. isolate CKG20 (GYM medium) showed the lowest chemical diversity of all prioritized bacterial extracts (Figure 6, Figure S4 and Table S6). Putatively identified compounds belonged to phenazine alkaloids $(44,48)$ and cyclic depsipeptides (51-53), of which the latter was the dominant chemical family in this extract. Cyclic depsipeptides were represented in three molecular clusters in the FBMN, since MS/MS analysis detected three different adduct types $\left([\mathrm{M}+\mathrm{H}]^{+},[\mathrm{M}+\mathrm{Na}]^{+},[\mathrm{M}+\mathrm{K}]^{+}\right)$that formed their own clusters due to their specific fragmentation patterns. In addition, the GNPS dereplication workflow annotated three compounds to known ubiquitous cell membrane components, i.e., glycerophospholipids $(42,45,50)$. The majority $(60 \%)$ of the detected metabolites and two molecular clusters did not match with any known compound.

Putatively identified compounds of Bacillus sp. extract CKG24-GYM were classified into three different chemical families, namely, isocoumarin derivatives $(\mathbf{5 5}, \mathbf{5 9}, \mathbf{6 0})$, polyketide glycosides (64), and various cyclic lipopeptides $(\mathbf{6 1 - 6 3 , 6 5 , 6 6 , 6 8 - 7 2 , 7 4 , 7 6 , 7 7 , 7 9 , 8 1 , 8 2 , 8 4 -}$ 87; Figure 6, Figure S5 and Table S7). In the FBMN, the dominance of cyclic lipopeptides was reflected by eight molecular clusters putatively annotated to this NP family. Putatively annotated cyclic lipopeptides can be further classified into bacillomycins (6163; $m / z$ 1071.5811-1099.6122 $\left.[\mathrm{M}+\mathrm{H}]^{+}\right)$, plipistatins $(65,66,68-72 ; m / z ~ 731.4171-753.4296$ $\left.[\mathrm{M}+2 \mathrm{H}]^{2+}\right)$, and surfactins $\left(\mathbf{7 4 , 7 6 , 7 7 , 7 9 , 8 1 , 8 2 , 8 4 - 8 7 ; ~} \mathrm{m} / z\right.$ 994.6426-1064.7209 $[\mathrm{M}+\mathrm{H}]^{+}$; Figure S5, Table S7). The putative assignment to three different subfamilies (bacillomycins, 
plipistatins, surfactins) and the detection of different adduct types (e.g., surfactins: $[\mathrm{M}+\mathrm{H}]^{+}$ and $[\mathrm{M}+\mathrm{Na}]^{+}$adducts) explain the formation of several distinct lipopeptide clusters. The highest annotation rate in this study $(71 \%)$ was achieved for this extract, i.e., only 10 compounds $(54,56-58,67,73,75,78,80,83)$ remained unannotated.

FBMN-based analysis also proved the high metabolite diversity (411 ions in total, 52 distinct molecular clusters) of the six selected fungal extracts (Figure 7). Putatively annotated clusters included alkaloids (indole and cytochalasan alkaloids), peptides (e.g., peptaibols), polyketides (e.g., macrolides), steroids (ergosterols), and terpenoids (meroand sesquiterpenoids). The largest molecular cluster, putatively annotated by GNPS and in silico dereplication workflows, is xanthone and zearalenone type polyketides. It contained metabolites produced by all three fungal strains. With the exception of this shared polyketide cluster, the chemical diversity of the selected strains differed. As an example, Trichoderma sp. showed 171-173 nodes and 24 exclusive clusters in the global network and was the most chemically diverse. Fusarium sp. and Penicillium sp. produced only eight and fifteen exclusive clusters, respectively.

Trichoderma sp. isolate CHG34 produced ergosterols, sorbicillinoid-type polyketides, and peptaibols (Figure 7, Figure S6 and Table S8), of which the latter dominated the metabolome. Various peptaibols such as trichokindins $(\mathbf{1 2 5}, \mathbf{1 4 3 , 1 4 4 , 1 4 7 , 1 5 0 , 1 5 1 , 1 5 4 , 1 5 5 , 1 5 7 )}$ and neoatroviridins $(\mathbf{1 4 9 , 1 5 6 , 1 6 0})$ were putatively annotated. As depicted in the FBMN (Figure S6), peptaibols are often detected as doubly charged (sodiated) ions $\left([\mathrm{M}+2 \mathrm{H}]^{2+}\right.$ and $\left.[\mathrm{M}+2 \mathrm{Na}]^{2+}\right)[35,36]$. Accordingly, several distinct peptaibol clusters such as trichokindins and neoatroviridins were annotated. Despite our integrated dereplication efforts, most compounds (73\%) in the Trichoderma extracts remained unannotated (Table S8). The amino acid sequence of two unannotated compounds, $m / z 770.5386[\mathrm{M}+\mathrm{H}]^{+}(\mathbf{1 0 3})$ and $m / z$ $754.5424[\mathrm{M}+\mathrm{H}]^{+}(\mathbf{1 0 5})$ (highlighted as "putatively novel lipopeptides" in the global MN; Figure 7 and Figure S6 and Table S8), were putatively predicted based on characteristic MS/MS fragments (Figure 8 and Figure S11). Tandem mass spectrometry (MS/MS) is an essential tool in peptide chemistry since mass differences of produced fragment ions allow the determination of the amino acid sequence of peptides [37]. Analysis of the MS/MS spectra of $\mathbf{1 0 3}$ and $\mathbf{1 0 5}$ revealed that both compounds contained seven amino acid residues. The first observed fragment of $\mathbf{1 0 3}(\mathrm{m} / \mathrm{z}$ value of 184.1341$)$ reflects the fatty acyl moiety Oc (octanoyl) connected to Gly $\left(\mathrm{C}_{10} \mathrm{H}_{18} \mathrm{NO}_{2}\right)$ at the $\mathrm{N}$-terminus of the putative peptide. The loss of $m / z 230.1994\left(\mathrm{C}_{12} \mathrm{H}_{26} \mathrm{~N}_{2} \mathrm{O}_{2}\right)$ positioned Leu/Ile-Leuol/Ileol at the C-terminus of $\mathbf{1 0 3}$ The full sequence of $103\left(\mathrm{~m} / z 770.5386[\mathrm{M}+\mathrm{H}]^{+}\right)$determined by MS/MS fragmentation was proposed as N-Oc-Gly-Gly-Leu/Ile-Val-Ser-Leu/Ile-Leuol/Ileol. The second putatively novel linear peptide $\left(\mathbf{1 0 5}, \mathrm{m} / z \mathbf{z} 54.5424[\mathrm{M}+\mathrm{H}]^{+}\right)$had a similar amino acid sequence as $\mathbf{1 0 3}$ but with Ser replaced by Ala. Accordingly, these two molecular ions may be novel linear seven-residue lipopeptides produced by Trichoderma sp. strain CHG34. The CAG and PDA extract of Trichoderma sp. CHG34 showed a high overlap of their chemical space (82\%) with 61 shared metabolites (Table S8).

In Fusarium sp. extracts CHG38-CAG and -PDA, the cyclic lipopeptide fusaristatin A (182) and chromone $(164,165)$, isocoumarin (166), naphthoquinone $(162,163,168)$, xanthone $(\mathbf{1 7 1}, \mathbf{1 7 8})$, and zearalenone $(\mathbf{1 6 9 , 1 7 6 , 1 7 9 )}$ polyketides were annotated (Figure 7 , Figure S7 and Table S9). According to the cluster analysis, xanthone and zearalenone polyketides dominated the FBMN by forming the two largest clusters. Zearalenone (176) was predicted by the GNPS-based MS/MS spectral match and guided us to putatively annotate 2'-hydroxyzearalanol (169) and zearalanone (179) in the same cluster. The majority of compounds (62\%) remained unidentified. Notably, cultivation of Fusarium sp. strain CHG38 triggered the production of several metabolites that were exclusive to either CAG (10 compounds) or PDA (16 compounds) medium. 

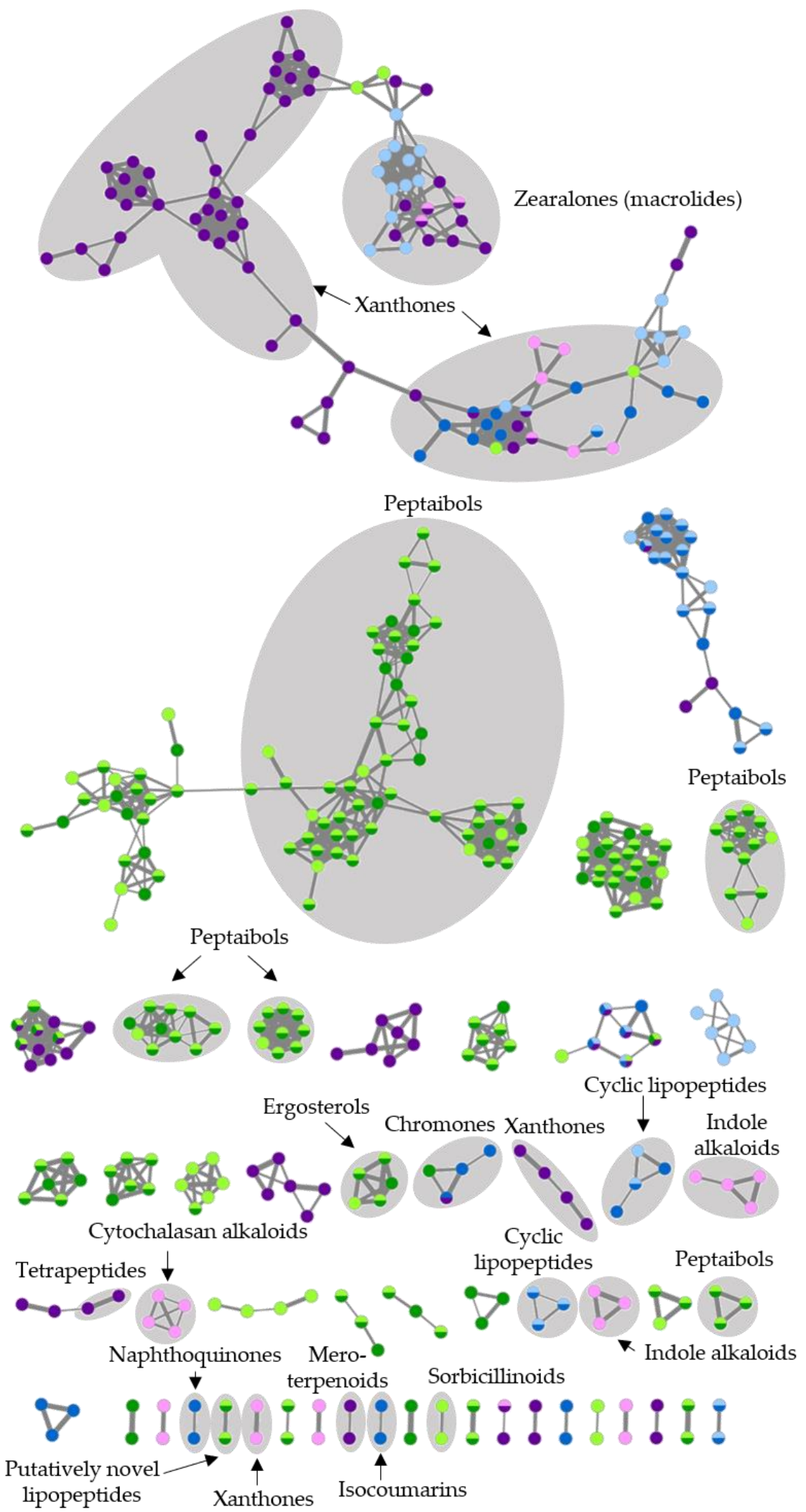

Figure 7. Global UPLC-MS/MS-based metabolome of six fungal extracts. The FBMN displays only molecular clusters containing $\geq 2$ nodes. The width of edges represents the cosine similarity between 2 nodes. Nodes are color-coded by the respective extract (CAG: light color; PDA: strong color): green: Trichoderma sp. strain CHG34; blue: Fusarium sp. strain CHG38; purple: Penicillium sp. strain CKG23. 


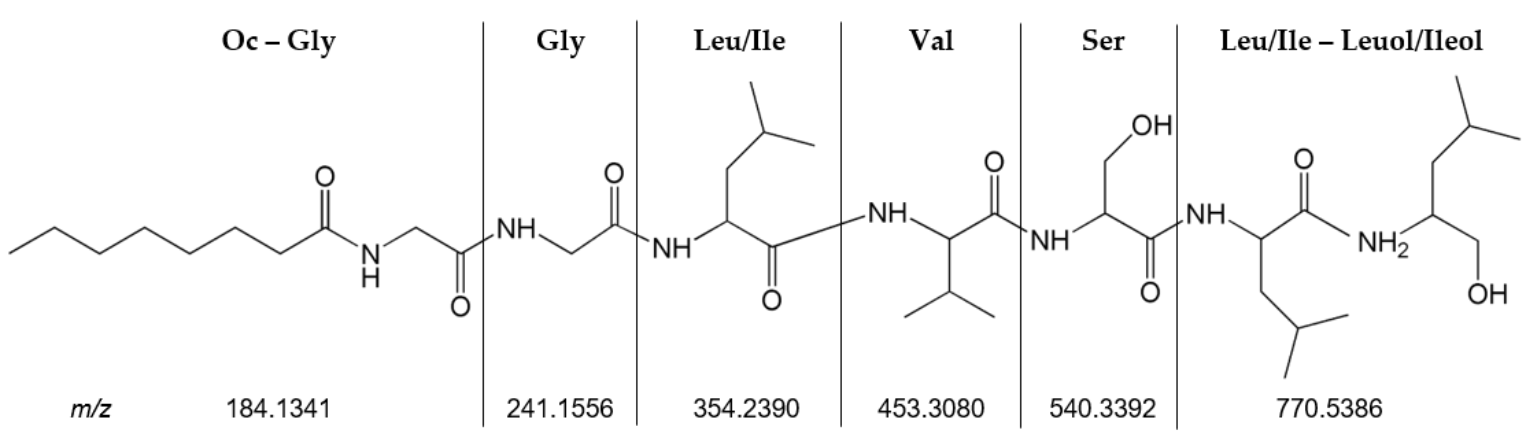

(a)<smiles>CCCCCCCC(=O)NCC(=O)NCC(=O)NC(CC(C)C)C(=O)C(NC(C(=O)NC(C)C(=O)NC(CC(C)C)C(N)=O)C(C)C)(C(=O)NC(CO)CC(C)C)C(C)C</smiles>

(b)

Figure 8. Two putatively novel seven-residue lipopeptides detected in Trichoderma sp. extracts CHG34-CAG and CHG34PDA. Structures were putatively predicted based on the experimentally determined MS/MS fragments for (a) compound $103\left(\mathrm{~m} / z 770.5386[\mathrm{M}+\mathrm{H}]^{+}\right)$and $(\mathbf{b})$ compound $105\left(\mathrm{~m} / z 754.5423[\mathrm{M}+\mathrm{H}]^{+}\right)$. Ala: alanine; Gly: glycine; Leu/Ile: (iso)leucine (leucine is displayed); Leuol/Ileol: (iso)leucinol (leucinol is displayed); Oc: octanoyl; Ser: serine; Val: valine.

The metabolome of Penicillium sp. strain CKG23 contained eight different chemical families, such as cytochalasans $(209,212)$, indole alkaloids $(206,219)$, meroterpenoids $(222)$, sesquiterpenoids (213), and various types of polyketides including zearalenone derivatives $(197,199,205,208,224 ;$ Figure 7 and Figure S8, Table S10). The largest cluster in the FBMN belonged to the polyketide family xanthones, which were also detected in Fusarium and Trichoderma sp. extracts in the global FBMN. Notably, the CAG and PDA extracts of Penicillium sp. strain CKG23 shared only seven molecular ions (Figure S12). Moreover, CKG23-PDA showed a strikingly higher chemical diversity with 185 detected peaks compared to CKG23CAG (51 peaks). For example, most putatively identified compounds in the zearalenone

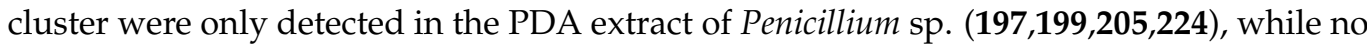
compound was exclusive to extract CKG23-CAG.

The untargeted metabolomics approach employed here revealed the huge chemical inventory of nine microbial extracts (Table 3). The metabolomes showed large variations between the different microbial taxa, but also the applied cultivation media impacted the chemical diversity. Compared to low annotation rates of $<2 \%$ normally achieved in untargeted metabolomics studies [38], the integrated dereplication effort applied herein significantly improved the putative annotation rates ranging from $24 \%$ (Streptomyces sp. extract CHG48-GYM) to 71\% (Bacillus sp. extract CKG24G). Nevertheless, many compounds and molecular clusters did not match any known compounds, suggesting that they could represent putatively new compounds. 
Table 3. Summary of the chemical inventory of nine selected crude extracts explored by untargeted FBMN-based metabolomics. Each extract is given with the number of nodes detected in the global bacterial (Figure 6) or fungal (Figure 7) FBMNs. In addition, putatively identified chemical families and annotation rates are indicated for each strain.

\begin{tabular}{cccc}
\hline Extract & Identification & Nodes & Putatively Annotated Chemical Families \\
\hline CHG48-GYM & Streptomyces sp. & 73 & Acetamides, diterpenoid glycosides, linear polyketides, nonactic \\
acid polyketides, oxazolidone alkaloids & Annotation Rate (\%) \\
CKG20-GYM & Micromonospora sp. & 61 & Cyclic depsipeptides, phenazine alkaloids, glycerophospholipids \\
CKG24-GYM & Bacillus sp. & 89 & Cyclic lipopeptides, isocoumarins, polyketide glycosides \\
CHG34-CAG & Trichoderma sp. & 173 & 24 \\
CHG34-PDA & Trichoderma sp. & 171 & Ergosterols, peptaibols, sorbicillinoids \\
CHG38-CAG & Fusarium sp. & 53 & Chromones, cyclic lipopeptides, isocoumarins, naphthoquinones, \\
CHG38-PDA & Fusarium sp. & 54 & xanthones, zearalenones \\
CKG23-CAG & Penicillium sp. & 29 & Cytochalasans, indole alkaloids, mero- and sesquiterpenoids, \\
CKG23-PDA & Penicillium sp. & 108 & styrylpyrones, tetrapeptides, xanthones, zearalenones \\
\hline
\end{tabular}

\section{Discussion}

In the present study, the unexplored gut microbiota of the tunicate $C$. intestinalis was investigated for its potential to deliver novel MNPs with pharmaceutical potential. The obtained strain collection represents with 101 gut-associated bacteria and fungi (Figure 1a, Table S1) the most comprehensive strain collection from the tunicate's gut available to date. A diverse microbial community (i.e., 33 different genera) was obtained from six different isolation media, which showed different suitability for growth of a diverse array of microorganisms (Figure 1b). For instance, glucose is a common carbon source for fungi [39], and, accordingly, most fungi were obtained from the glucose-containing media, WSP and PDA. In addition, media containing several carbon sources and other complex compounds often yield the highest microbial diversity [18], and this is in line with our finding that most isolates were obtained from the complex WSP medium (glucose, malt extract, peptone, and yeast extract). This indicates that the selection of isolation media has a huge impact on the isolated microbiota due to specific nutrient requirements of different microorganisms [18,19]. Furthermore, other cultivation conditions such as temperature $\left(22^{\circ} \mathrm{C}\right)$ also significantly influence the cultivable fraction of bacteria and fungi [18,19].

Although highly abundant bacterial genera such Shewanella and Vibrio and fungal genera such as Penicillium and Trichoderma (Figure 2) were previously isolated from the gut of Ciona spp. [23,26-28], most microbial genera were isolated for the first time from the gut of $C$. intestinalis. The high abundance of Gammaproteobacteria is in accordance with a previous culture-independent study performed on the gut microbiome of Ciona spp. [29]. We have recently described the cultivable microbiota of the tunic of C. intestinalis [31] that differed strikingly from the gut-associated microbial community isolated herein. Both tissues shared only few microbial genera (e.g., bacteria: Bacillus and Vibrio; fungi: Fusarium and Penicillium), which is in accordance with culture-independent microbiome studies on the gut and tunic of Ciona spp., indicating tissue-specific microbial communities [29,30]. Moreover, the diversity of culture-dependent fungi was higher in the gut (gut: 40 isolates assigned to 20 genera; tunic: 22 isolates, 15 genera), while bacteria were more prominent in the tunic (89 isolates assigned 37 genera; gut: 61 isolates, 13 genera) [31]. In line with our previous study on the tunic-associated microbiota [31], the gut-associated microbiota, especially the bacterial community, was more diverse in Kiel than in Helgoland samples (Figures 1 and 2). This may be attributed to different salinity levels (Kiel: brackish, Helgoland: oceanic salinity) and the comparably higher anthropogenic input, i.e., more eutrophic conditions, at the sampling site in Kiel Fjord [31]. Moreover, samples were obtained from different depths (Helgoland: <1 m depth; Kiel: approx. $3 \mathrm{~m} \mathrm{depth)}$ and different artificial surfaces (Helgoland: pontoon; Kiel: mussel-cultivation basket), which both may have influenced the obtained microbial diversity. Other parameters not determined in this study, e.g., diet, water temperature, and the age and genetic background of the sampled specimens, may be additional factors shaping the diversity of the cultivable microbiota $[18,40,41]$. 
Gut-derived microbial extracts $(n=103)$ were screened against a panel of cancer cell lines and microbial pathogens, since ascidians and their associated microorganisms are well-known producers of MNPs with antimicrobial and anticancer properties [11,13,42]. The antimicrobial assays included the so-called ESKAPE panel, drug-resistant bacterial pathogens that were categorized by the WHO as priority level 1 and 2 for the discovery of new antimicrobial agents [43]. Most extracts $(n=65)$ exhibited activity against MRSA and/or E. faecium, but also anticancer $(n=23)$ and antifungal $(n=11)$ activities were observed (Figure 3, Table S2), exceeding bioactivity levels previously reported for cultivable bacteria associated with solitary ascidians $[44,45]$. The high rate of bioactivity observed in this study is in line with the excellent biodiscovery potential reported for tunicates and their microbial associates [11,13,42]. As outlined before, an intact gut microbiota has crucial functions for the health and performance of its host [1,3]. Possible functions related to chemical defense and nutrition were already proposed for cultivable bacteria obtained from the intestine of a solitary ascidian and a sea urchin [46,47]. The high proportion of microorganisms with, e.g., antibacterial properties (64\%) indicates their potential involvement in the tunicate's chemical defense. However, it was beyond the scope of this study to detect specific functions fulfilled by specific cultivable gut-associated microorganisms in the host-microbiota interplay.

Bioactive crude extracts $(n=68)$ were subjected to a two-step selection procedure considering the bioactivity profile and chemical diversity of the extracts to prioritize the most promising extracts for in-depth metabolome mining. This strategy aided prioritization of nine microbial extracts affiliated to the bacterial genera Bacillus, Micromonospora, and Streptomyces as well as the fungal genera Fusarium, Penicillium, and Trichoderma (Figures 4 and 5). These microbial genera are known as the most talented producers of MNPs highlighting the strength of the applied prioritization pipeline (e.g., $[9,48,49])$.

The integrated dereplication approach combining automated and manual dereplication tools allowed the putative identification of 94 metabolites (Tables S5-S10). They belonged to various NP classes such as alkaloids, lipids, peptides, polyketides, steroids, and terpenoids, revealing a huge metabolic capacity of the prioritized microbial strains. Dereplication was substantially supported by the recently released FBMN workflow [32], which, in combination with other tools, led to annotation rates of up to $71 \%$. Increasing annotation rates in untargeted metabolomic experiments is crucial to overcome time-consuming re-isolation of known compounds, which severely hampers biodiscovery efforts [33].

Untargeted metabolomics studies on fungi (e.g., Fusarium and Penicillium spp. [31,50,51]) and bacteria (e.g., Streptomyces and Salinispora spp. [52]) cultured on the same medium already revealed huge chemical variations at both species and strain level and are therefore used as chemotaxonomic species discrimination markers ([51] and references therein). Hence, we expected to find distinct chemical profiles of strains from the same genus, e.g., Penicillium or Streptomyces (Figure 4, Figure 5 and Figure S1, Tables S3-S4). Beyond this, variations in metabolite diversity in different media is also a well-known phenomenon in the OSMAC (one strain-many compounds) approach [53]. In line with this, Fusarium sp. strain CHG38 and Penicillium sp. strain CKG23 showed differential bioactivities and metabolomes when cultured on two different media (Figure 7, Figure S7-S8 and S12, Table 1, Table 2 and Tables S9-S10). Furthermore, the fungal cultivation media CAG and PDA appeared to trigger the production of various (bioactive) metabolites, e.g., the metabolomes of Fusarium sp. CHG38 and Penicillium sp. CKG23 were richer when cultured on PDA medium, while cultivation of Trichoderma sp. on CAG yielded more compounds (Figure 7, Tables S8-S10). Both media contain the simple sugar glucose, an ideal carbon source for fungal growth [39], plus an additional mixed carbon source, which seemingly meets requirements for fungal growth and production of secondary metabolites. In contrast, GYM and MB media used for bacterial strains were not equally suitable as only one bacterial extract obtained from cultivation on MB medium met the bioactivity selection criterion (P. anguilliseptica extract CKG38-MB), while seven GYM extracts exerted strong antimicrobial and/or anticancer activities (Table S2). While MB medium mimics the major 
mineral composition of seawater, GYM medium lacks these minerals, but contains glucose and malt extract, which are easily accessible carbon sources. However, eleven bacterial strains, including all Vibrio sp. isolates, failed to grow on GYM medium. This may be attributed to the lack of sodium chloride $(\mathrm{NaCl})$ in this medium, since most Vibrio spp. require $\mathrm{NaCl}$ for growth [54]. Apart from the media composition, other parameters such as the temperature, solid regime (in contrast to liquid cultures) or aeration may have influenced the observed chemical composition [53,55]. Moreover, artificial laboratory conditions often lack important environmental cues such as multispecies interactions, which can lead to, e.g., silencing of important biosynthetic gene clusters [55]. Therefore, the obtained metabolomic compositions may not necessarily reflect the true metabolite repertoire of the organism [55].

The observed anti-MRSA and E. faecium activity of Streptomyces sp. extract CHG48 (Table 1) might be explained by the diterpenoid glycoside platensimycin B (6) and two nonactic acid polyketides $(\mathbf{1 8}, \mathbf{3 3})$, all with reported antibacterial activities [56-59]. The putatively annotated polyketide homononactyl homononactate (18) shows weak activity against colon cancer cell line HCT116 [60], but not against lung cancer cell line A549 [56]. Hence, this compound cannot explain the detected selective anticancer activities against cell lines A549 and A375 (Table 2). Moreover, none of the dereplicated metabolites can explain the detected antifungal activities of Streptomyces sp. extract CHG48-GYM against C. albicans and C. neoformans (Table 1), and the annotation rate was the lowest detected in this study (24\%; Table S5). We therefore consider Streptomyces sp. isolate CHG48 as a promising candidate strain for isolation of its chemical constituents.

The antibacterial and anticancer activities (Tables 1 and 2) of Micromonospora sp. (CKG20-GYM) may be attributed to the putatively annotated cyclic depsipeptides rakicidins (51-53) [61-63] and the phenazine alkaloid diazepinomicin (44) [49,64] (Figure 6 and Figure S4, Table S6), for which these activities are known. About 60\% of the detected compounds remained unannotated, including a putatively novel rakicidin derivative (49), which is worth investigating in future studies.

The Bacillus sp. extract CKG24-GYM inhibited the growth of microbial pathogens and showed antiproliferative activity against cancer cell lines A375, A549 and HCT116 (Tables 1 and 2). Anti-MRSA activity has been described for the putatively identified isocoumarin derivative amicoumacin-A (55) [65] and the polyketide glycoside aurantinin B (64) [66]. Inhibitory activities against colon cancer cell line HCT116 are reported for some surfactinlike lipopeptides $\mathbf{( 7 9 , 8 2 , 8 4 , 8 6 )}$ [67]. Bacillomycins (61-63) [68] and some cyclic lipopeptides $(\mathbf{6 5}, \mathbf{6 6}, \mathbf{6 8}-\mathbf{7 2})$ reportedly show antifungal properties [69] possibly relating to the observed fungicidal activity of CKG24-GYM. In combination with the high annotation rate (71\%), these results render Bacillus sp. extract CKG24-GYM not favorable for future studies.

Among the fungi, Trichoderma spp. are the major source of peptaibols [70,71] and, accordingly, extracts of Trichoderma sp. strain CHG34 were dominated by this NP class (Figure 7 and Figure S6, Table S8). Moderate anticancer activities were observed in both Trichoderma sp. extracts (CHG34-CAG, -PDA; Table 2), which may originate from the putatively identified peptaibols neoatroviridin B-D $(\mathbf{1 4 9 , 1 5 6 , 1 6 0 )}[72]$. Antibacterial activity was only detected in the PDA extract of Trichoderma sp. strain CHG34 (Table 1), but none of the compounds exclusive to this extract $(\mathbf{9 0}, \mathbf{9 2}, \mathbf{1 1 1})$ could be putatively identified as annotation rates remained low for Trichoderma sp. strain CHG34 (27\%). Two new lipopeptide structures $(\mathbf{1 0 3}, \mathbf{1 0 5})$ were proposed based on the predicted amino acid sequences from the product ions. Future efforts will encompass isolation and confirmation of the putative structures and also exploration of this strain for other novel MNPs for the treatment of infectious diseases.

As outlined above, Fusarium sp. extracts showed different bioactivities and metabolomes (Figure 7, Figure S7, Table 1, Table 2 and Table S9). The antimicrobial and anticancer properties of Fusarium sp. extract CHG38-PDA may originate from the putatively annotated xanthone derivative griseoxanthone $C(\mathbf{1 7 8})[73,74]$ and the naphthoquinone norjavanicin (163) [75]. In addition, the shared metabolite fusaristatin A (182) reportedly inhibits the 
proliferation of lung cancer cells [76]. Fusarium spp. are also prominent producers of mycotoxins, including the macrolide zearalenone (176) detected in this study [77]. Zearalenone (176) shows antifungal but no antibacterial activities [78]. Hence, antibacterial activities of Fusarium sp. extract CHG38-CAG remain unresolved, since none of its putatively annotated compounds have been shown to possess antibacterial activities. Accordingly, extract CHG38-CAG is highlighted for future chemical investigations.

Detailed LC-MS/MS analyses of the Penicillium sp. isolate CKG23 revealed distinct chemical profiles of its CAG and PDA extracts (Figure 5a, Figure 7, Figures S8 and S12, Table S10). Anticancer activities of CKG23-CAG may be attributed to the putatively identified indole alkaloid communesin B (219) and the cytochalasan chaetoglobosin A (212) that were shown to inhibit the proliferation of cancer cell lines A549 $(\mathbf{2 1 2}, \mathbf{2 1 9})$ and HCT116 (219) $[79,80]$. In addition, the meroterpenoid andrastin A (222) putatively annotated in CKG23-CAG is a farnesyltransferase inhibitor that prevents correct functioning of, e.g., RAS proteins (common oncogenes), rendering it a promising anticancer lead compound [81]. Antiproliferative activity of CKG23-PDA and observed antibacterial activities (including Gram-negative pathogens) of both Penicillium sp. extracts could not be linked to any of the dereplicated compounds. Moreover, extract CKG23-PDA had the most distinct chemistry of all Penicillium sp. extracts (Figure 5a), indicating that the PDA extract of Penicillium sp. CKG23 is worth pursuing in future studies.

In this study, we showed that the cultivable fraction of the gut-associated microbiota of $C$. intestinalis is diverse and specific. The majority of the yet unexplored gut-associated cultivable microorganisms showed antimicrobial and/or anticancer activities, suggesting a significant potential for discovery of new drug leads. Computationally assisted metabolome mining of nine prioritized bioactive crude extracts led to the putative identification of 94 metabolites assigned to 24 different chemical families. Nevertheless, most compounds and molecular clusters remained unannotated and may therefore be novel MNPs. Several extracts containing putatively novel and bioactive metabolites were highlighted, in particular Streptomyces extract CHG48-CAG, Trichoderma sp. extracts CHG34CAG and CHG34-PDA, Fusarium sp. extract CHG38-CAG, and Penicillium sp. extract CKG23-PDA. This emphasizes the benefit of working with marine microorganisms obtained from previously unexplored sources and is in line with the strongly increasing contribution of microorganisms in MNP discovery [9]. It is widely accepted that MNPs have higher success rates in drug discovery compared to their terrestrial counterparts [82]. Being evolved and prevalidated by natural pressures and adaptation processes for millions of years, NPs represent "privileged scaffolds in drug discovery" [83], whose bioactivity and structural diversity surpass any synthetic compound prepared in the laboratory [84]. To conclude, the obtained cultivable gut-associated microbial community of C. intestinalis delivered several promising microorganisms that seem to harbor an unexplored chemical space. Putatively novel compounds and their bioactivities need to be verified in future isolation and structure elucidation studies.

\section{Materials and Methods}

\subsection{Sampling and Isolation of Microorganisms}

Ciona intestinalis was sampled in September 2017 at two locations, the German North Sea island Helgoland ( Germany, 54.177102, 7.893053) and Kiel Fjord (Kiel, Germany, Baltic Sea, 54.382062, 10.162059). Samples were collected in local harbors from a pontoon (Helgoland, $<1 \mathrm{~m}$ depth) or from a mussel cultivation basket (Kiel Fjord, approximately $3 \mathrm{~m}$ depth). Dissection and inoculation of microbiological samples were conducted at the same day.

Six solid media (1.8\% agar) were used to isolate a diverse bacterial and fungal community from the ascidian's gut: two C. intestinalis media adjusted to Baltic (CB) and North Sea salinity (CN; [31]), MB (3.74\% Marine Broth 2216), potato dextrose agar (PDA [85]), TSB ( $0.3 \%$ trypticase soy broth, $1 \%$ sodium chloride) and modified Wickerham medium (WSP [86]). Agar (bacteriology grade) and sodium chloride were purchased from Ap- 
pliChem (Darmstadt, Germany), malt extract, Marine Broth, and trypticase soy broth from Becton Dickinson (Sparks, MD, USA), glucose, peptone (from soymeal), and granulated yeast extract from Merck (Darmstadt, Germany), and potato infusion powder was ordered at Sigma Aldrich (Steinheim, Germany).

The gut ( $n=2$ biological replicates per sampling site) was carefully dissected and placed into a sterile $1.5 \mathrm{~mL}$ reaction tube. The dissected gut was diluted 1:1 with sterile artificial seawater $(1.8 \%(\mathrm{~K})$ or $3 \%(\mathrm{H})$ Instant ocean, Blacksburg, VA, USA) and gently homogenized with a sterile pestle. Agar media were inoculated with $100 \mu \mathrm{L}$ aliquots and their 1:10 and 1:100 dilutions. Petri dishes were checked for growth of fungi and bacteria after incubation for one and three weeks in the dark at $22{ }^{\circ} \mathrm{C}$. Microbial colonies showing distinct macroscopic phenotypes were transferred to fresh agar plates until pure cultures were obtained. Microbial strains were cryo-conserved at $-80^{\circ} \mathrm{C}$ using the ready-to-use Microbank $^{\mathrm{TM}}$ system (Richmond Hill, ON, Pro Lab Diagnostics, Canada).

\subsection{DNA Extraction and Identification of Microbial Isolates}

DNA was extracted by applying a freeze and thaw protocol (bacteria) or by mechanical lysis (fungi) as described elsewhere [31,87]. If subsequent PCR amplification of the target fragment failed, DNA extraction was repeated with the DNeasy Plant Mini Kit (Qiagen, Hilden, Germany) according to the manufacturer's instructions. Some modifications of the protocol were applied as previously described [31].

Molecular identification was performed following established protocols for amplification of the 16S rRNA gene (bacteria) or the ITS1-5.8S-ITS2 region (fungi) [87]. The 18S and $28 \mathrm{~S}$ rRNA gene were additionally amplified for fungal strains with ambiguous identification (for protocols see [88,89]). PCR conditions for amplifying the large ribosomal subunit of the rRNA were modified as previously described [31]. Sanger sequencing [90] of successfully amplified DNA fragments was conducted at LGC Genomics GmbH (Berlin, Germany). FASTA files of quality checked and trimmed DNA sequences were searched against the NCBI (National Center for Biotechnology Information) nucleotide database using BLAST (Basic Local Alignment Search Tool [91]). One bacterial isolate (CKG60) could only be identified at family level. Application of Naive Bayesian rRNA Classifier v2.11 of the Ribosomal Database Project using the 16S rRNA training set at a 95\% confidence threshold [92] resulted in identification of this isolate to genus level. Bacterial and fungal DNA sequences were deposited in GenBank under the accession numbers MW065489549 (gut-associated bacteria), MW064137-74 (gut-associated fungi, ITS), and MW064175-6 (gut-associated fungi, 18S; Table S1).

\subsection{Cultivation and Extraction of Gut-Associated Microbial Strains}

Out of 101 gut-derived microbial isolates, 29 were excluded from cultivation due to laboratory safety concerns based on the Technical Rules for Biological Agents (TRBA 460, TRBA 466). Fifteen additional strains were excluded, since they were affiliated to the same species as another strain isolated from the same sampling site, which led to the final selection of 27 bacterial and 30 fungal strains for cultivation $(n=57$; Table S2). Microbial isolates were cultured in duplicate (i.e., two biological replicates) on two different media. Bacterial isolates were cultured on the solid agar media glucose-yeast-malt (GYM [93]) and MB. Solid casamino acids-glucose (CAG [94]) and PDA media were used for growing fungal strains. Ingredients not listed in Section 4.1. were purchased from Carl Roth (Karlsruhe, Germany). CAG, GYM and PDA were chosen due to their excellent potential to trigger production of novel and bioactive compounds (see [31] and references therein). $\mathrm{MB}$ medium was chosen to ensure sufficient growth of all bacterial isolates. For cultivation of precultures, solid media were inoculated with cryo-conservation beads and incubated in the dark at $22{ }^{\circ} \mathrm{C}$ until the agar was completely overgrown. Main cultures were inoculated by transferring microbial colonies or an overgrown piece of agar with an inoculation loop to the respective agar medium. Fungi were inoculated on five agar plates and bacteria on ten plates in parallel. As outlined above, each strain was cultivated on two respective media 
in duplicate yielding 20 (fungi) or 40 (bacteria) petri dishes per strain. Microbial cultures were grown in the dark for seven (bacteria) or 21 (fungi) days at $22{ }^{\circ} \mathrm{C}$. Eleven bacterial strains did not grow on GYM medium, hence were only cultivated on MB medium.

For solvent extractions, the agar was cut with a flat spatula and mixed with $200 \mathrm{~mL}$ (fungi) or $400 \mathrm{~mL}$ (bacteria) ethyl acetate (EtOAc; VWR International, Leuven, Belgium) in a glass bottle. The mixture was homogenized with a T25 basic Ultra Turrax (IKA-Werke, Staufen, Germany; $30 \mathrm{~s}$ at 13,000 rpm), which was followed by maceration overnight $\left(120 \mathrm{rpm}, 22^{\circ} \mathrm{C}\right)$. The solvent was decanted and washed with an equal volume of ultrapurified water to remove salts (Arium Lab water systems, Sartorius, Goettingen, Germany) in a liquid-liquid partitioning experiment. The EtOAc phase was transferred to a round bottom flask. The extraction process was repeated in the same manner, with the only exception that the extraction process was reduced to $15 \mathrm{~min}$ in an ultrasonic bath. Combined EtOAc extracts were evaporated to dryness and re-solubilized in $4 \mathrm{~mL}$ methanol $(\mathrm{MeOH}$; ULC-MS grade, Biosolve Chimie, Dieuze, France). Extracts were filtered through a $0.2 \mu \mathrm{m}$ PTFE (polytetrafluoroethylene) filter (VWR International, Darmstadt, Germany), dried again in pre-weighed vials to determine their extract weight, and stored at $-20^{\circ} \mathrm{C}$. Media blanks were prepared as controls by using the same protocol.

\subsection{Biological Assays}

Dried crude extracts were dissolved in dimethyl sulfoxide (Carl Roth) at a concentration of $20 \mathrm{mg} / \mathrm{mL}$. Microbial pathogens included the ESKAPE panel (Enterococcus faecium, Efm, DSM 20477; methicillin-resistant Staphylococcus aureus, MRSA, DSM 18827; Klebsiella pneumoniae, Kp, DSM 30104; Acinetobacter baumannii, Ab, DSM 30007; Pseudomonas aeruginosa, Psa, DSM 1128; Escherichia coli, Ec, DSM 1576), as well as the pathogenic fungi Candida albicans (Ca, DSM 1386) and Cryptococcus neoformans (Ca, DSM 6973). The following four cancer cell lines were selected for anticancer assays: A375 (malignant melanoma cell line), A549 (lung carcinoma cell line), HCT116 (colon cancer cell line), and MDA-MB231 (human breast cancer line). Test organisms and cell lines were purchased from Leibniz Institute DSMZ-German Collection of Microorganisms and Cell Cultures (Braunschweig, Germany) and CLS Cell Lines Service (Eppelheim, Germany). Tests were performed in 96-well plates at a final concentration of $100 \mu \mathrm{g} / \mathrm{mL}$ as previously described $[95,96]$. For each extract, its two biological replicates were tested in duplicate each (i.e., two technical replicates). The antibiotics amphotericin (Cn), ampicillin (Efm), chloramphenicol (MRSA, Ec, Kp), doxycycline $(\mathrm{Ab})$, nystatin $(\mathrm{Ca})$, polymyxin $\mathrm{B}(\mathrm{Psa})$, and the cytostatic agent doxorubicin (cancer cell lines) served as positive controls. Prioritized extracts were additionally subjected to $\mathrm{IC}_{50}$ determinations (also two biological and two technical replicates) by applying a previously published protocol [95].

\subsection{Metabolomic Analyses \\ 4.5.1. UPLC-QToF-MS/MS Measurements}

All solvents used for UPLC-based metabolomic analyses were ordered at Biosolve Chimie or LGC Standards (Wesel, Germany) in ULC-MS grade. Crude extracts (two biological replicates each) re-dissolved in $\mathrm{MeOH}$ (final concentration of $1.0 \mathrm{mg} / \mathrm{mL}$ ) were measured on an Acquity UPLC I-Class System connected to a Xevo G2-XS QToF Mass Spectrometer (Waters, Milford, MA, USA). Crude extracts were injected $(0.3 \mu \mathrm{L})$ and separated on an Acquity UPLC HSS T3 column (High Strength Silica C18, $1.8 \mu \mathrm{m}, 2.1 \times 100 \mathrm{~mm}$, Waters) operating at $40{ }^{\circ} \mathrm{C}$. The binary mobile phase consisted of ultra-purified water (A) and acetonitrile (B), both spiked with $0.1 \%$ formic acid. The elution gradient pumped at a flow rate of $0.6 \mathrm{~mL} / \mathrm{min}$ was as follows (\% of A given): initial, $99 \%$; $11.5 \mathrm{~min}, 1 \% ; 14.5 \mathrm{~min}$, $1 \%$; $14.5-16$ min 99\%. LC-MS chromatograms and MS/MS fragmentation spectra were recorded as previously described [31]. The same settings were applied to analyze $\mathrm{MeOH}$ (solvent control) and media blanks. 


\subsubsection{Pre-Processing of UPLC-MS/MS Data and Statistics}

The ProteoWizard tool msconvert 3.0.20010 was used to transform acquired spectra to mzXML format [97]. Quality filtering and removal of media and contaminant peaks were carried out in MZmine 2.53 [98]. Briefly, mass lists were compiled for compounds with a retention time $\left(R_{t}\right)$ between 1 and 12 min with an intensity above 30,000 (MS) or 50 (MS/MS). Chromatograms were built at a minimum peak height of 60,000 and an $\mathrm{m} / \mathrm{z}$ tolerance of $0.005 \mathrm{Da}$ or $15 \mathrm{ppm}$. Deconvolution of chromatograms was performed with the baseline cut-off algorithm using the same noise level and peak height as above. Isotope grouping and alignment of peaks was performed with an $\mathrm{m} / \mathrm{z}$ tolerance of $0.001 \mathrm{Da}$ or $10 \mathrm{ppm}$ and an $\mathrm{R}_{\mathrm{t}}$ tolerance of $0.5 \mathrm{~min}$. The alignment of peaks was conducted with the join aligner method by using an $m / z$ to $\boldsymbol{R}_{\mathbf{t}}$ ratio of 75:25. Finally, peak lists were filtered with an $\mathrm{m} / \mathrm{z}$ range of 150-1200 Da. Media and solvent control peaks were detected using the same approach (noise level: 1000; peak height: 3000) and subsequently removed from the filtered peak list. Filtered peak lists were subjected to statistical analyses to assess the chemical distinctiveness of the microbial extracts. Therefore, PCoA plots reflecting the metabolomic (dis)similarities of the selected extracts and ANOSIM scores (Euclidean distance) were calculated in Past v3.12 [99].

\subsubsection{Feature-Based Molecular Networking and Dereplication}

Processed MS/MS data of prioritized extracts (see Section 2.3.) were submitted in MGF format to the FBMN workflow [32] available at the open access platform GNPS [34]. FBMNs were compiled as previously described [31] and visualized with Cytoscape v3.7.1 [100].

Compounds showing distinct peaks in the LC-MS chromatograms above the noise threshold were subjected to a dereplication workflow combining automated and manual dereplication tools. The prediction of putative molecular formulae was performed in MassLynx v4.1 (Waters). Four NP databases, i.e., Dictionary of Natural Products ( http://dnp.chemnetbase.com), MarinLit (http://pubs.rsc.org/marinlit/), The Natural Products Atlas (https:/ / www.npatlas.org/joomla/index.php/search/basic-search [101]) and Reaxys (https:/ / www.reaxys.com), were inspected for putative hits for the predicted molecular formulae. In parallel, pre-processed MS/MS data were subjected to the dereplication workflow of GNPS [34]. The same dataset was also automatically dereplicated by applying the in silico MS/MS database of the Universal Natural Product Database [33]. Putatively annotated NPs were verified by comparing the biological sources, $\mathrm{R}_{\mathrm{t}}$, and MS/MS spectra (if detected), of which the latter was aided by the in-silico prediction tool CFM-ID 3.0 [102].

Supplementary Materials: The following are available online at https:/ / www.mdpi.com/1660-339 7/19/1/6/s1, Figure S1: Venn diagram of exclusive and shared peaks of N. prasina and Streptomyces sp. extracts. Figure S2: Structures of putatively identified compounds. Figures S3-S8: FBMNs of prioritized crude extracts. Figures S9-S11: MS/MS spectra of putatively annotated compounds. Figure S12: Comparative metabolome analyses of Penicillium sp. isolate CKG23. Table S1: Taxonomic classification of microbial strains isolated from the gut of $C$. intestinalis sampled in Helgoland and Kiel Fjord. Table S2: Antimicrobial and anticancer activities of microbial crude extracts. Tables S3-S4: Statistical comparison of chemically distinct microbial crude extracts. Tables S5-S10: Putatively identified compounds in prioritized microbial crude extracts.

Author Contributions: Conceptualization, C.U., M.B., D.T.; data curation, C.U., V.A.E., E.O.-D.; formal analysis, C.U., E.O.-D.; writing—original draft, C.U., M.B., D.T.; writing—review and editing, C.U., M.B., D.T. All authors have read and agreed to the published version of the manuscript.

Funding: This research received no external funding.

Institutional Review Board Statement: Not applicable.

Informed Consent Statement: Not applicable.

Data Availability Statement: The sequencing data presented in this study are openly available in GenBank at NCBI (accession numbers: MW065489-549, MW064137-74, MW064175-6). The metabolomics data presented in this study are available on request from the corresponding au- 
thor. The data are not publicly available due to ongoing research on the novel chemistry of the presented microbial strains.

Acknowledgments: We kindly acknowledge the Biological Institute Helgoland (BAH) of the Alfred Wegener Institute (AWI), Helmholtz Centre for Polar and Marine Research, for access to laboratories and the associated Centre of Scientific Diving for sample collection. Sampling in Kiel was assisted by Kieler Meeresfarm UG. We thank Hilger Jagau and Christiane Schulz for assistance during microbiological work. Jana Heumann and Arlette Wenzel-Storjohann are thanked for performing the biological assays. Larissa Buedenbender, Pradeep Dewapriya, and Florent Magot are acknowledged for support during UPLC-QToF-MS/MS-measurements and dereplication. We thank Antje Labes for helpful discussions throughout the project.

Conflicts of Interest: The authors declare no conflict of interest.

\section{References}

1. Lozupone, C.A.; Stombaugh, J.I.; Gordon, J.I.; Jansson, J.K.; Knight, R. Diversity, stability and resilience of the human gut microbiota. Nature 2012, 489, 220-230. [CrossRef] [PubMed]

2. Dishaw, L.J.; Cannon, J.P.; Litman, G.W.; Parker, W. Immune-directed support of rich microbial communities in the gut has ancient roots. Dev. Comp. Immunol. 2014, 47, 36-51. [CrossRef]

3. Fraune, S.; Anton-Erxleben, F.; Augustin, R.; Franzenburg, S.; Knop, M.; Schröder, K.; Willoweit-Ohl, D.; Bosch, T.C.G. Bacteriabacteria interactions within the microbiota of the ancestral metazoan Hydra contribute to fungal resistance. ISME J. 2014, 9, 1543-1556. [CrossRef] [PubMed]

4. Pickard, J.M.; Zeng, M.Y.; Caruso, R.; Nunez, G. Gut microbiota: Role in pathogen colonization, immune responses, and inflammatory disease. Immunol. Rev. 2017, 279, 70-89. [CrossRef] [PubMed]

5. Westerdahl, A.; Olsson, J.C.; Kjelleberg, S.; Conway, P.L. Isolation and characterization of turbot (Scophtalmus maximus)-associated bacteria with inhibitory effects against Vibrio anguillarum. Appl. Environ. Microbiol. 1991, 57, 2223-2228. [CrossRef] [PubMed]

6. Sugita, H.; Matsuo, N.; Hirose, Y.; Iwato, M.; Deguchi, Y. Vibrio sp. strain NM 10, isolated from the intestine of a Japanese coastal fish, has an inhibitory effect against Pasteurella piscicida. Appl. Environ. Microbiol. 1997, 63, 4986-4989. [CrossRef]

7. Sawabe, T.; Oda, Y.; Shiomi, Y.; Ezura, Y. Alginate degradation by bacteria isolated from the gut of sea urchins and abalones. Microb. Ecol. 1995, 30, 193-202. [CrossRef]

8. Kobayashi, J.; Ishibashi, M. Bioactive metabolites of symbiotic marine microorganisms. Chem. Rev. 1993, 93, 1753-1769. [CrossRef]

9. Carroll, A.R.; Copp, B.R.; Davis, R.A.; Keyzers, R.A.; Prinsep, M.R. Marine natural products. Nat. Prod. Rep. 2020, 37, 175-223. [CrossRef]

10. Martins, A.; Vieira, H.; Gaspar, H.; Santos, S. Marketed marine natural products in the pharmaceutical and cosmeceutical industries: Tips for success. Mar. Drugs 2014, 12, 1066-1101. [CrossRef]

11. Chen, L.; Hu, J.S.; Xu, J.L.; Shao, C.L.; Wang, G.Y. Biological and chemical diversity of ascidian-associated microorganisms. Mar. Drugs 2018, 16, 362. [CrossRef]

12. Thomas, T.R.A.; Kavlekar, D.P.; LokaBharathi, P.A. Marine drugs from sponge-microbe association-A review. Mar. Drugs 2010, 8, 1417-1468. [CrossRef] [PubMed]

13. Casertano, M.; Menna, M.; Imperatore, C. The ascidian-derived metabolites with antimicrobial properties. Antibiotics 2020, 9, 510. [CrossRef] [PubMed]

14. Rath, C.M.; Janto, B.; Earl, J.; Ahmed, A.; Hu, F.Z.; Hiller, L.; Dahlgren, M.; Kreft, R.; Yu, F.; Wolff, J.J.; et al. Meta-omic characterization of the marine invertebrate microbial consortium that produces the chemotherapeutic natural product ET-743. ACS Chem. Biol. 2011, 6, 1244-1256. [CrossRef] [PubMed]

15. Liu, Y.; Zhao, S.; Ding, W.; Wang, P.; Yang, X.; Xu, J. Methylthio-aspochalasins from a marine-derived fungus Aspergillus sp. Mar. Drugs 2014, 12, 5124-5131. [CrossRef]

16. Li, X.; Zhao, Z.; Ding, W.; Ye, B.; Wang, P.; Xu, J. Aspochalazine A, a novel polycyclic aspochalasin from the fungus Aspergillus sp. Z4. Tetrahedron Lett. 2017, 58, 2405-2408. [CrossRef]

17. Xu, Y.; Huang, R.; Liu, H.; Yan, T.; Ding, W.; Jiang, Y.; Wang, P.; Zheng, D.; Xu, J. New polyketides from the marine-derived fungus Letendraea sp. 5XNZ4-2. Mar. Drugs 2019, 18, 18. [CrossRef]

18. Alain, K.; Querellou, J. Cultivating the uncultured: Limits, advances and future challenges. Extremophiles 2009, 13, 583-594. [CrossRef]

19. Garza, D.R.; Dutilh, B.E. From cultured to uncultured genome sequences: Metagenomics and modeling microbial ecosystems. Cell. Mol. Life Sci. 2015, 72, 4287-4308. [CrossRef]

20. Tamaki, H.; Sekiguchi, Y.; Hanada, S.; Nakamura, K.; Nomura, N.; Matsumura, M.; Kamagata, Y. Comparative analysis of bacterial diversity in freshwater sediment of a shallow eutrophic lake by molecular and improved cultivation-based techniques. Appl. Environ. Microbiol. 2005, 71, 2162-2169. [CrossRef]

21. Chen, L.; Fu, C.; Wang, G. Microbial diversity associated with ascidians: A review of research methods and application. Symbiosis 2017, 71, 19-26. [CrossRef] 
22. Carver, C.E.; Mallet, A.L.; Vercaemer, B. Biological synopsis of the solitary tunicate Ciona intestinalis. Can. Man. Rep. Fish. Aquat. Sci. 2006, 2746, 1-55.

23. Dishaw, L.J.; Flores-Torres, J.A.; Mueller, M.G.; Karrer, C.R.; Skapura, D.P.; Melillo, D.; Zucchetti, I.; De Santis, R.; Pinto, M.R.; Litman, G.W. A Basal chordate model for studies of gut microbial immune interactions. Front. Immunol. 2012, 3, 96. [CrossRef] [PubMed]

24. Leigh, B.A.; Liberti, A.; Dishaw, L.J. Generation of germ-free Ciona intestinalis for studies of gut-microbe interactions. Front. Microbiol. 2016, 7, 2092. [CrossRef] [PubMed]

25. Franchi, N.; Ballarin, L. Immunity in Protochordates: The tunicate perspective. Front. Immunol. 2017, 8, 674. [CrossRef]

26. Dishaw, L.J.; Leigh, B.; Cannon, J.P.; Liberti, A.; Mueller, M.G.; Skapura, D.P.; Karrer, C.R.; Pinto, M.R.; De Santis, R.; Litman, G.W. Gut immunity in a protochordate involves a secreted immunoglobulin-type mediator binding host chitin and bacteria. Nat. Commun. 2016, 7, 10617. [CrossRef]

27. Leigh, B.; Karrer, C.; Cannon, J.P.; Breitbart, M.; Dishaw, L.J. Isolation and characterization of a Shewanella phage-host system from the gut of the tunicate, Ciona intestinalis. Viruses 2017, 9, 60. [CrossRef]

28. Liberti, A.; Cannon, J.P.; Litman, G.W.; Dishaw, L.J. A soluble immune effector binds both fungi and bacteria via separate functional domains. Front. Immunol. 2019, 10, 369. [CrossRef]

29. Dishaw, L.J.; Flores-Torres, J.; Lax, S.; Gemayel, K.; Leigh, B.; Melillo, D.; Mueller, M.G.; Natale, L.; Zucchetti, I.; De Santis, R.; et al. The gut of geographically disparate Ciona intestinalis harbors a core microbiota. PLoS ONE 2014, 9, e93386. [CrossRef]

30. Blasiak, L.C.; Zinder, S.H.; Buckley, D.H.; Hill, R.T. Bacterial diversity associated with the tunic of the model chordate Ciona intestinalis. ISME J. 2014, 8, 309-320. [CrossRef]

31. Utermann, C.; Echelmeyer, V.A.; Blümel, M.; Tasdemir, D. Culture-dependent microbiome of the Ciona intestinalis tunic: Isolation, bioactivity profiling and untargeted metabolomics. Microorganisms 2020, 8, 1732. [CrossRef] [PubMed]

32. Nothias, L.F.; Petras, D.; Schmid, R.; Duhrkop, K.; Rainer, J.; Sarvepalli, A.; Protsyuk, I.; Ernst, M.; Tsugawa, H.; Fleischauer, M.; et al. Feature-based molecular networking in the GNPS analysis environment. Nat. Methods 2020, 17, 905-908. [CrossRef] [PubMed]

33. Allard, P.M.; Peresse, T.; Bisson, J.; Gindro, K.; Marcourt, L.; Pham, V.C.; Roussi, F.; Litaudon, M.; Wolfender, J.L. Integration of molecular networking and in-silico MS/MS fragmentation for natural products dereplication. Anal. Chem. 2016, 88, 3317-3323. [CrossRef] [PubMed]

34. Wang, M.; Carver, J.J.; Phelan, V.V.; Sanchez, L.M.; Garg, N.; Peng, Y.; Nguyen, D.D.; Watrous, J.; Kapono, C.A.; Luzzatto-Knaan, T.; et al. Sharing and community curation of mass spectrometry data with Global Natural Products Social Molecular Networking Nat. Biotechnol. 2016, 34, 828-837. [CrossRef] [PubMed]

35. Poirier, L.; Montagu, M.; Landreau, A.; Mohamed-Benkada, M.; Grovel, O.; Sallenave-Namont, C.; Biard, J.F.; Amiard-Triquet, C.; Amiard, J.C.; Pouchus, Y.F. Peptaibols: Stable markers of fungal development in the marine environment. Chem. Biodivers. 2007, 4, 1116-1128. [CrossRef] [PubMed]

36. Marik, T.; Tyagi, C.; Balazs, D.; Urban, P.; Szepesi, A.; Bakacsy, L.; Endre, G.; Rakk, D.; Szekeres, A.; Andersson, M.A.; et al. Structural diversity and bioactivities of peptaibol compounds from the Longibrachiatum Clade of the filamentous fungal fenus Trichoderma. Front. Microbiol. 2019, 10, 1434. [CrossRef]

37. Paizs, B.; Suhai, S. Fragmentation pathways of protonated peptides. Mass Spectrom. Rev. 2005, 24, 508-548. [CrossRef]

38. Da Silva, R.R.; Dorrestein, P.C.; Quinn, R.A. Illuminating the dark matter in metabolomics. Proc. Natl. Acad. Sci. USA 2015, 112, 12549-12550. [CrossRef]

39. De Oliveira Costa, O.; Nahas, E. Growth and enzymatic responses of phytopathogenic fungi to glucose in culture media and soil. Braz. J. Microbiol. 2012, 43, 332-340. [CrossRef]

40. Goddard-Dwyer, M.; Lopez-Legentil, S.; Erwin, P.M. Microbiome variability across the native and invasive range of the ascidian Clavelina oblonga. Appl. Environ. Microbiol. 2020. AEM.02233-20. [CrossRef]

41. Giatsis, C.; Sipkema, D.; Smidt, H.; Verreth, J.; Verdegem, M. The colonization dynamics of the gut microbiota in tilapia larvae. PLoS ONE 2014, 9, e103641. [CrossRef] [PubMed]

42. Menna, M. Antitumor potential of natural products from Mediterranean ascidians. Phytochem. Rev. 2009, 8, 461-472. [CrossRef]

43. Global Priority List of Antibiotic-Resistant Bacteria to Guide Research, Discovery, and Development of New Antibiotics (World Health Organization 2017). Available online: https://www.who.int/medicines/publications/global-priority-list-antibioticresistant-bacteria/en/ (accessed on 19 October 2020).

44. Chen, L.; Wang, X.-N.; Fu, C.-M.; Wang, G.-Y. Phylogenetic analysis and screening of antimicrobial and antiproliferative activities of culturable bacteria associated with the ascidian Styela clava from the Yellow Sea, China. Biomed. Res. Int. 2019, $2019,1-14$. [CrossRef] [PubMed]

45. Romanenko, L.A.; Kalinovskaya, N.I.; Mikhailov, V.V. Taxonomic composition and biological activity of microorganisms associated with a marine ascidian Halocynthia aurantium. Russ. J. Mar. Biol. 2001, 27, 291-296. [CrossRef]

46. Meziti, A.; Kormas, K.A.; Pancucci-Papadopoulou, M.A.; Thessalou-Legaki, M. Bacterial phylotypes associated with the digestive tract of the sea urchin Paracentrotus lividus and the ascidian Microcosmus sp. Russ. J. Mar. Biol. 2007, 33, 84-91. [CrossRef]

47. Laport, M.S.; Bauwens, M.; Collard, M.; George, I. Phylogeny and antagonistic activities of culturable bacteria associated with the gut microbiota of the sea urchin (Paracentrotus lividus). Curr. Microbiol. 2018, 75, 359-367. [CrossRef] 
48. Agrawal, S.; Acharya, D.; Adholeya, A.; Barrow, C.J.; Deshmukh, S.K. Nonribosomal peptides from marine microbes and their antimicrobial and anticancer potential. Front. Pharmacol. 2017, 8, 828. [CrossRef]

49. Hifnawy, M.S.; Fouda, M.M.; Sayed, A.M.; Mohammed, R.; Hassan, H.M.; AbouZid, S.F.; Rateb, M.E.; Keller, A.; Adamek, M.; Ziemert, N.; et al. The genus Micromonospora as a model microorganism for bioactive natural product discovery. RSC Adv. 2020, 10, 20939-20959. [CrossRef]

50. Macia-Vicente, J.G.; Shi, Y.N.; Cheikh-Ali, Z.; Grun, P.; Glynou, K.; Kia, S.H.; Piepenbring, M.; Bode, H.B. Metabolomics-based chemotaxonomy of root endophytic fungi for natural products discovery. Environ. Microbiol. 2018, 20, 1253-1270. [CrossRef]

51. Oppong-Danquah, E.; Passaretti, C.; Chianese, O.; Blümel, M.; Tasdemir, D. Mining the metabolome and the agricultural and pharmaceutical potential of sea foam-derived fungi. Mar. Drugs 2020, 18, 128. [CrossRef]

52. Crüsemann, M.; O’Neill, E.C.; Larson, C.B.; Melnik, A.V.; Floros, D.J.; da Silva, R.R.; Jensen, P.R.; Dorrestein, P.C.; Moore, B.S. Prioritizing natural product diversity in a collection of 146 bacterial strains based on growth and extraction protocols. J. Nat. Prod. 2016, 80, 588-597. [CrossRef] [PubMed]

53. Bode, H.B.; Bethe, B.; Hofs, R.; Zeeck, A. Big effects from small changes: Possible ways to explore nature's chemical diversity. Chembiochem 2002, 3, 619-627. [CrossRef]

54. Tison, D.L.; Kelly, M.T. Vibrio species of medical importance. Diagn. Microbiol. Infect. Dis. 1984, 2, 263-276. [CrossRef]

55. Romano, S.; Jackson, S.A.; Patry, S.; Dobson, A.D.W. Extending the "One Strain Many Compounds" (OSMAC) principle to marine microorganisms. Mar. Drugs 2018, 16, 244. [CrossRef] [PubMed]

56. Huang, H.; Lan, X.; Wang, Y.; Tian, L.; Fang, Y.; Zhang, L.; Zhang, K.; Zheng, X. New bioactive derivatives of nonactic acid from the marine Streptomyces griseus derived from the plant Salicornia sp. Phytochem. Lett. 2015, 12, 190-195. [CrossRef]

57. Jeong, S.Y.; Shin, H.J.; Kim, T.S.; Lee, H.S.; Park, S.K.; Kim, H.M. Streptokordin, a new cytotoxic compound of the methylpyridine class from a marine-derived Streptomyces sp. KORDI-3238. J. Antibiot. 2006, 59, 234-240. [CrossRef] [PubMed]

58. Kusche, B.R.; Smith, A.E.; McGuirl, M.A.; Priestley, N.D. Alternating pattern of stereochemistry in the nonactin macrocycle is required for antibacterial activity and efficient ion binding. J. Am. Chem. Soc. 2009, 131, 17155-17165. [CrossRef]

59. Zhang, C.; Ondeyka, J.; Guan, Z.; Dietrich, L.; Burgess, B.; Wang, J.; Singh, S.B. Isolation, structure and biological activities of platensimycin B4 from Streptomyces platensis. J. Antibiot. 2009, 62, 699-702. [CrossRef]

60. Han, L.; Huo, P.; Chen, H.; Li, S.; Jiang, Y.; Li, L.; Xu, L.; Jiang, C.; Huang, X. New derivatives of nonactic and homononactic acids from Bacillus pumilus derived from Breynia fruticosa. Chem. Biodivers. 2014, 11, 1088-1098. [CrossRef]

61. Oku, N.; Matoba, S.; Yamazaki, Y.M.; Shimasaki, R.; Miyanaga, S.; Igarashi, Y. Complete stereochemistry and preliminary structureactivity relationship of rakicidin A, a hypoxia-selective cytotoxin from Micromonospora sp. J. Nat. Prod. 2014, 77, 2561-2565. [CrossRef]

62. Yamazaki, Y.; Kunimoto, S.; Ikeda, D. Rakicidin A: A hypoxia-selective cytotoxin. Biol. Pharm. Bull. 2007, 30, 261-265. [CrossRef] [PubMed]

63. Landwehr, W.; Karwehl, S.; Schupp, P.J.; Schumann, P.; Wink, J. Biological active rakicidins A, B and E produced by the marine Micromonospora sp. isolate Guam1582. Adv. Biotechnol. Microbiol. 2016, 1, 1-5. [CrossRef]

64. Charan, R.D.; Schlingmann, G.; Janso, J.; Bernan, V.; Feng, X.; Carter, G.T. Diazepinomicin, a new antimicrobial alkaloid from a marine Micromonospora sp. J. Nat. Prod. 2004, 67, 1431-1433. [CrossRef] [PubMed]

65. Itoh, J.; Omoto, S.; Shomura, T.; Nishizawa, N.; Miyado, S.; Yuda, Y.; Shibata, U.; Inouye, S. Amicoumacin-A, a new antibiotic with strong antiinflammatory and antiulcer activity. J. Antibiot. 1981, 34, 611-613. [CrossRef] [PubMed]

66. Konda, Y.; Nakagawa, A.; Harigaya, Y.; Onda, M.; Masuma, R.; Omura, S. Aurantinin B, a new antimicrobial antibiotic from bacterial origin. J. Antibiot. 1988, 41, 268-270. [CrossRef]

67. Zhuravleva, O.I.; Afiyatullov, S.S.; Ermakova, S.P.; Nedashkovskaya, O.I.; Dmitrenok, P.S.; Denisenko, V.A.; Kuznetsova, T.A. New C14-surfactin methyl ester from the marine bacterium Bacillus pumilus KMM 456. Russ. Chem. Bull. 2011, 59, $2137-2142$. [CrossRef]

68. Mhammedi, A.; Peypoux, F.; Besson, F.; Michel, G. Bacillomycin F, a new antibiotic of iturin group: Isolation and characterization. J. Antibiot. 1982, 35, 306-311. [CrossRef]

69. Kimura, K.; Nakayama, S.; Nakamura, J.; Takada, T.; Yoshihama, M.; Esumi, Y.; Itoh, Y.; Uramoto, M. SNA-60-367, new peptide enzyme inhibitors against aromatase. J. Antibiot. 1997, 50, 529-531. [CrossRef]

70. Mohamed-Benkada, M.; Montagu, M.; Biard, J.F.; Mondeguer, F.; Verite, P.; Dalgalarrondo, M.; Bissett, J.; Pouchus, Y.F. New short peptaibols from a marine Trichoderma strain. Rapid Commun. Mass Spectrom. 2006, 20, 1176-1180. [CrossRef]

71. Mukherjee, P.K.; Wiest, A.; Ruiz, N.; Keightley, A.; Moran-Diez, M.E.; McCluskey, K.; Pouchus, Y.F.; Kenerley, C.M. Two classes of new peptaibols are synthesized by a single non-ribosomal peptide synthetase of Trichoderma virens. J. Biol. Chem. 2011, 286, 4544-4554. [CrossRef]

72. Oh, S.U.; Yun, B.S.; Lee, S.J.; Kim, J.H.; Yoo, I.D. Atroviridins A-C and neoatroviridins A-D, novel peptaibol antibiotics produced by Trichoderma atroviride F80317. I. Taxonomy, fermentation, isolation and biological activities. J. Antibiot. 2002, 55, 557-564. [CrossRef] [PubMed]

73. Hawas, U.W.; Farrag, A.R.H.; Ahmed, E.F.; Abou El-Kassem, L.T. Cytotoxic effect of Fusarium equiseti fungus metabolites against N-Nitrosodiethylamine- and CCL4-induced hepatocarcinogenesis in rats. Pharm. Chem. J. 2018, 52, 326-333. [CrossRef] 
74. Wang, Q.X.; Bao, L.; Yang, X.L.; Guo, H.; Yang, R.N.; Ren, B.; Zhang, L.X.; Dai, H.Q.; Guo, L.D.; Liu, H.W. Polyketides with antimicrobial activity from the solid culture of an endolichenic fungus Ulocladium sp. Fitoterapia 2012, 83, 209-214. [CrossRef] [PubMed]

75. Holenstein, J.; DÉFago, G. Inheritance of naphthazarin production and pathogenicity to pea in Nectria haematococca. J. Exp. Bot. 1983, 34, 927-935. [CrossRef]

76. Shiono, Y.; Tsuchinari, M.; Shimanuki, K.; Miyajima, T.; Murayama, T.; Koseki, T.; Laatsch, H.; Funakoshi, T.; Takanami, K.; Suzuki, K. Fusaristatins A and B, two new cyclic lipopeptides from an endophytic Fusarium sp. J. Antibiot. 2007, 60, 309-316. [CrossRef]

77. Desjardins, A.E. Fusarium Mycotoxins: Chemistry, Genetics, and Biology; American Phytopathological Society (APS Press): St. Paul, MN, USA, 2006.

78. Utermark, J.; Karlovsky, P. Role of zearalenone lactonase in protection of Gliocladium roseum from fungitoxic effects of the mycotoxin zearalenone. Appl. Environ. Microbiol. 2007, 73, 637-642. [CrossRef]

79. Pompeo, M.M.; Cheah, J.H.; Movassaghi, M. Total synthesis and anti-cancer activity of all known communesin alkaloids and related derivatives. J. Am. Chem. Soc. 2019, 141, 14411-14420. [CrossRef]

80. Huang, S.; Chen, H.; Li, W.; Zhu, X.; Ding, W.; Li, C. Bioactive chaetoglobosins from the mangrove endophytic fungus Penicillium chrysogenum. Mar. Drugs 2016, 14, 172. [CrossRef]

81. Nielsen, K.F.; Dalsgaard, P.W.; Smedsgaard, J.; Larsen, T.O. Andrastins A-D, Penicillium roqueforti metabolites consistently produced in blue-mold-ripened cheese. J. Agr. Food Chem. 2005, 53, 2908-2913. [CrossRef]

82. Sigwart, J.D.; Blasiak, R.; Jaspars, M.; Jouffray, J.-B.; Tasdemir, D. Unlocking the potential of marine biodiscovery. Nat. Prod. Rep. 2021. [CrossRef]

83. Davison, E.K.; Brimble, M.A. Natural product derived privileged scaffolds in drug discovery. Curr. Opin. Chem. Biol. 2019, 52, 1-8. [CrossRef] [PubMed]

84. Newman, D.J.; Cragg, G.M. Natural products as sources of new drugs over the nearly four decades from 01/1981 to $09 / 2019$. J. Nat. Prod. 2020, 83, 770-803. [CrossRef] [PubMed]

85. Oppong-Danquah, E.; Parrot, D.; Blümel, M.; Labes, A.; Tasdemir, D. Molecular networking-based metabolome and bioactivity analyses of marine-adapted fungi co-cultivated with phytopathogens. Front. Microbiol. 2018, 9, 1-20. [CrossRef] [PubMed]

86. Silber, J.; Ohlendorf, B.; Labes, A.; Erhard, A.; Imhoff, J.F. Calcarides A-E, antibacterial macrocyclic and linear polyesters from a Calcarisporium strain. Mar. Drugs 2013, 11, 3309-3323. [CrossRef] [PubMed]

87. Utermann, C.; Parrot, D.; Breusing, C.; Stuckas, H.; Staufenberger, T.; Blümel, M.; Labes, A.; Tasdemir, D. Combined genotyping, microbial diversity and metabolite profiling studies on farmed Mytilus spp. from Kiel Fjord. Sci. Rep. 2018, 8, 7983. [CrossRef] [PubMed]

88. Gomes, N.C.; Fagbola, O.; Costa, R.; Rumjanek, N.G.; Buchner, A.; Mendona-Hagler, L.; Smalla, K. Dynamics of fungal communities in bulk and maize rhizosphere soil in the tropics. Appl. Environ. Microbiol. 2003, 69, 3758-3766. [CrossRef]

89. Hopple, J.S., Jr.; Vilgalys, R. Phylogenetic relationships in the mushroom genus Coprinus and dark-spored allies based on sequence data from the nuclear gene coding for the large ribosomal subunit RNA: Divergent domains, outgroups, and monophyly. Mol. Phylogenet. Evol. 1999, 13, 1-19. [CrossRef]

90. Sanger, F.; Nicklen, S.; Coulson, A.R. DNA sequencing with chain-terminating inhibitors. Proc. Natl. Acad. Sci. USA 1977, 74, 5463-5467. [CrossRef]

91. Altschul, S.F.; Gish, W.; Miller, W.; Myers, E.W.; Lipman, D.J. Basic local alignment search tool. J. Mol. Biol. 1990, 215 , 403-410. [CrossRef]

92. Wang, Q.; Garrity, G.M.; Tiedje, J.M.; Cole, J.R. Naive Bayesian classifier for rapid assignment of rRNA sequences into the new bacterial taxonomy. Appl. Environ. Microbiol. 2007, 73, 5261-5267. [CrossRef]

93. Wu, B.; Wiese, J.; Labes, A.; Kramer, A.; Schmaljohann, R.; Imhoff, J.F. Lindgomycin, an unusual antibiotic polyketide from a marine fungus of the Lindgomycetaceae. Mar. Drugs 2015, 13, 4617-4632. [CrossRef] [PubMed]

94. Stevens, R.B. Mycology Guidebook; University of Washington Press: Seattle, WA, USA, 1974; p. 682.

95. Pfeifer Barbosa, A.L.; Wenzel-Storjohann, A.; Barbosa, J.D.; Zidorn, C.; Peifer, C.; Tasdemir, D.; Cicek, S.S. Antimicrobial and cytotoxic effects of the Copaifera reticulata oleoresin and its main diterpene acids. J. Ethnopharmacol. 2019, 233, 94-100. [CrossRef] [PubMed]

96. Petersen, L.E.; Marner, M.; Labes, A.; Tasdemir, D. Rapid metabolome and bioactivity profiling of fungi associated with the leaf and rhizosphere of the Baltic seagrass Zostera marina. Mar. Drugs 2019, 17, 419. [CrossRef] [PubMed]

97. Chambers, M.C.; Maclean, B.; Burke, R.; Amodei, D.; Ruderman, D.L.; Neumann, S.; Gatto, L.; Fischer, B.; Pratt, B.; Egertson, J.; et al. A cross-platform toolkit for mass spectrometry and proteomics. Nat. Biotechnol. 2012, 30, 918-920. [CrossRef]

98. Pluskal, T.; Castillo, S.; Villar-Briones, A.; Oresic, M. MZmine 2: Modular framework for processing, visualizing, and analyzing mass spectrometry-based molecular profile data. BMC Bioinform. 2010, 11, 395. [CrossRef]

99. Hammer, Ø.; Harper, D.A.T.; Ryan, P.D. PAST: Paleontological statistics software package for education and data analysis. Palaeontol. Electron. 2001, 4, 1-9.

100. Shannon, P.; Markiel, A.; Ozier, O.; Baliga, N.S.; Wang, J.T.; Ramage, D.; Amin, N.; Schwikowski, B.; Ideker, T. Cytoscape: A software environment for integrated models of biomolecular interaction networks. Genome Res. 2003, 13, 2498-2504. [CrossRef] 
101. van Santen, J.A.; Jacob, G.; Singh, A.L.; Aniebok, V.; Balunas, M.J.; Bunsko, D.; Neto, F.C.; Castaño-Espriu, L.; Chang, C.; Clark, T.N.; et al. The Natural Products Atlas: An open access knowledge base for microbial natural products discovery. ACS Cent. Sci. 2019, 5, 1824-1833. [CrossRef]

102. Djoumbou-Feunang, Y.; Pon, A.; Karu, N.; Zheng, J.; Li, C.; Arndt, D.; Gautam, M.; Allen, F.; Wishart, D.S. CFM-ID 3.0: Significantly improved ESI-MS/MS prediction and compound identification. Metabolites 2019, 9, 72. [CrossRef] 